Article

\title{
Optimal Placement, Sizing and Coordination of FACTS Devices in Transmission Network Using Whale Optimization Algorithm
}

\author{
Muhammad Nadeem ${ }^{1}$, Kashif Imran 1,*(D), Abraiz Khattak ${ }^{1}$, Abasin Ulasyar ${ }^{1}$, Anamitra Pal ${ }^{2}$, \\ Muhammad Zulqarnain Zeb ${ }^{1}$, Atif Naveed Khan ${ }^{1}$ and Malhar Padhee ${ }^{2}$ \\ 1 US Pakistan Center for Advance Studies in Energy (USPCAS-E), National University of Sciences and \\ Technology (NUST), H-12, Islamabad 44000, Pakistan; nkmeraj@gmail.com (M.N.); \\ abraiz@uspcase.nust.edu.pk (A.K.); abasin@uspcase.nust.edu.pk (A.U.); zulqarnainzeb@gmail.com (M.Z.Z.); \\ atifnaveedkhan@yahoo.com (A.N.K.) \\ 2 School of Electrical Computer and Energy Engineering, Arizona State University, Tempe, AZ 85287, USA; \\ anamitra.pal@asu.edu (A.P.); mpadhee@asu.edu (M.P.) \\ * Correspondence: kashifimran@uspcase.nust.edu.pk
}

Received: 13 December 2019; Accepted: 7 January 2020; Published: 8 February 2020

check for updates

\begin{abstract}
Flexible AC Transmission Systems (FACTS) play an important role in minimizing power losses and voltage deviations while increasing the real power transfer capacity of transmission lines. The extent to which these devices can provide benefits to the transmission network depend on their optimal location and sizing. However, finding appropriate locations and sizes of these devices in an electrical network is difficult since it is a nonlinear problem. This paper proposes a technique for the optimal placement and sizing of FACTS, namely the Thyristor-Controlled Series Compensators (TCSCs), Shunt VARs Compensators (SVCs), and Unified Power Flows Controllers (UPFCs). To find the optimal locations of these devices in a network, weak buses and lines are determined by constructing PV curves of load buses, and through the line stability index. Then, the whale optimization algorithm (WOA) is employed not only to find an ideal ratings for these devices but also the optimal coordination of SVC, TCSC, and UPFC with the reactive power sources already present in the network (tap settings of transformers and reactive power from generators). The objective here is the minimization of the operating cost of the system that consists of active power losses and FACTS devices cost. The proposed method is applied to the IEEE 14 and 30 bus systems. The presented technique is also compared with Genetic Algorithm (GA) and Particle Swarm Optimization (PSO). The findings showed that total system operating costs and transmission line losses were considerably reduced by WOA as compared to existing metaheuristic optimization techniques.
\end{abstract}

Keywords: FACTS; line stability index $\left(\mathrm{L}_{\mathrm{mn}}\right)$; PV curves; whale optimization algorithm (WOA)

\section{Introduction}

Electrical power generating stations are usually located far away from load centers, and utilities greatly rely on existing generation to satisfy load demand via power export-import arrangements. Therefore, practical power systems are highly interconnected. Due to excessive interconnections, network restructuring, and dynamic load patterns, some transmission lines operate well above their thermal and stability limits [1]. The voltage profile of the power system gets affected by the uneven loading of transmission lines that leads to an unstable power system. 


\subsection{Stability Issues of Power Transmission}

Power system stability is usually described as the capability of the electrical system to remain in synchronism and continue its operation following a disturbance. The recent increase in the requirement of electrical energy and participation of private electricity producers has made the electric power industry very competitive. Therefore, utilities are more interested in transferring a large amount of power optimally through the existing network to gain more revenue instead of expanding the transmission system because of economic and environmental constraints.

Active power flow between two busses with voltages $V_{1}$ and $V_{2}$ and having respective angles is given by Equation (1) [1].

$$
P=\frac{V_{1} V_{2}}{X} \sin \delta
$$

From Equation (1), real power flow can be increased by increasing bus voltages and angles differences or by decreasing reactance of the line. However, voltages of buses cannot be increased arbitrarily as they should be within $5 \%$ of the nominal voltages. Bus angles differences are also typically restricted below $35^{\circ}$ [2]. If we consider $V_{1}=V_{2}=V$, the total power that can be transmitted on a lossless transmission line is determined by the reactance of the line. Hence, line reactance often sets the theoretical steady-state limit on power transmission. The thermal limit for practical transmission lines with resistance $R$ may be set by $I^{2} R$ losses, which cause heating in a conductor. Thus, it changes the physical characteristics of the transmission line, and at a certain temperature, it may cause permanent sag. Usually, for large transmission lines reactance $(X)$, and for small transmission line resistance $(R)$, sets the thermal limits. However, transmission line reactance cannot be decreased significantly because of thermal limitations [2,3]. In addition, there are limitations imposed on transmission lines due to its insulation capabilities, which are referred to as dielectric limits. Total power transfer capability of transmission lines can also be restricted by stability limitations, which is because of voltage or frequency collapse, steady-state stability, sub-synchronous resonance, and transient stability. From the above discussion, it can be concluded that the loading capability of the transmission line has mainly three restrictions: stability limits, thermal limits, and dielectric limits.

For short transmission lines, the maximum loading capabilities can be determined by thermal limits of conductor [3], which in turn depend on the load current, atmosphere conditions, and electrical and thermal characteristics of the conductor. So, one way to increase thermal limit of transmission lines is to upgrade the existing structure by changing the conductor to a larger current rating. Another solution is to expand the capacity of a transmission line by converting a single circuit to a double circuit. However, these solutions are not feasible because of budget and problems associated with finding the appropriate right of way. For these reasons, utilities are mainly interested in utilizing the existing network optimally to transfer maximum power instead of expanding the existing power system. Conventionally, electro-mechanical equipment was used to compensate the line and to increase its power transferring capability. However, these devices have the disadvantage that they give high switching transients. Compensations are accomplished only in a step-by-step manner, and due to their mechanical nature, they cannot be initiated frequently. So, compensators built on solid-state technology are required.

Flexible AC Transmission Systems (FACTS) devices play a key role these days to enhance voltage stability, power transmission capacity and to decrease transmission line congestions [4-9]. Technical comparisons of FACTS are discussed in [10]. The selection of the type of FACTS mainly depends on its intended use. A series compensator like TCSC controls the impedance of line in which it is connected while a parallel compensator, e.g., SVC, controls reactive power at its point of connection. A series-shunt controller like UPFC can control both system quantities [10].

\subsection{Controlled Parameters of Different FACTS}

Each FACTS device controls its respective parameters to increase the overall efficiency of the power system. Control parameters of some commonly used FACTS devices are presented in Figure 1. 
A comparison of some basic types of FACTS devices on the basis of their impact on power system is presented in Table 1 [11].

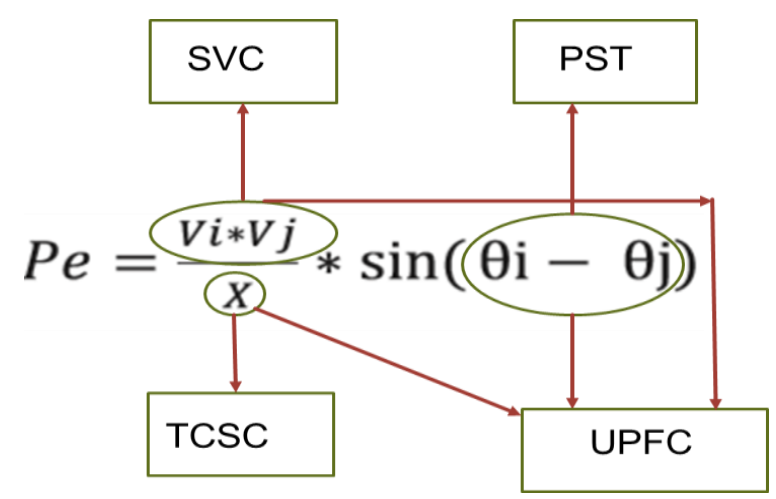

Figure 1. Control parameters of commonly used FACTS devices.

Table 1. Comparison of basic types of FACTS devices based on their impact on different applications.

\begin{tabular}{ccccc}
\hline FACTS Device & $\begin{array}{c}\text { Real Power } \\
\text { Flow }\end{array}$ & $\begin{array}{c}\text { Transient } \\
\text { Stability }\end{array}$ & $\begin{array}{c}\text { Voltage } \\
\text { Control }\end{array}$ & $\begin{array}{c}\text { Dynamic } \\
\text { Stability }\end{array}$ \\
\hline $\begin{array}{c}\text { Thyristor-controlled series-compensators } \\
\text { (TSSC, TCSC) }\end{array}$ & Medium & Strong & Small & Medium \\
$\begin{array}{c}\text { Static synchronous compensators } \\
\text { (STATCOM) }\end{array}$ & Small & Medium & Strong & Medium \\
$\begin{array}{c}\text { Static-VAR-Compensators } \\
\text { (TCS, SVC, TRS) }\end{array}$ & Small & Small & Strong & Medium \\
$\begin{array}{c}\text { Unified-Power-Flow-Controllers } \\
\text { (UPFC) }\end{array}$ & Strong & Medium & Strong & Medium \\
\hline
\end{tabular}

It can be seen from Table 1 that UPFC can be a strong candidate for all the mentioned applications. However, the cost of installation of FACTS should be considered before choosing the type of FACTS to be installed. The installation cost of the UPFC is higher than the SVC, hence if only voltage stability is required then SVC can be a better option than UPFC.

While placing FACTS controllers in power systems, some common questions arise, where is the best location to install FACTS? What sort of FACTS should be placed? How much capacity of FACTS is required? In this framework, numerous authors presented their own approaches. For example, genetic algorithm (GA) was utilized for the optimal placement of SVC, TCSC, and UPFC in [4]; however, their optimal mix of quantities in the power system network was not discussed. In [12], an artificial algae algorithm (AAA) was proposed for optimal allocation of UPFC to reduce transmission line congestion, but optimal coordination of UPFC with other reactive power sources was not discussed. Non-dominated Sorted genetic algorithm (NSGA-II) was used in [13] to determine sizing, numbers, and locations for TCSCs. Its main objective was to enhance load-ability of the power system; however, placing TCSC and their corresponding effect on already present reactive power sources in the system was not present. An interesting study using the Marginal Pricing Index (MPI) and Marginal Congestion Index (MCI) was done in [14] where an optimal allocation of TCSC, SVC, UPFC, and wind farms were discussed together. Their utilization for power network risk mitigation and assessment in a centralized power market is also presented. Another study was done in [15] for the combine siting, sizing, and operation of FACTS and wind farms, devices used were TCSC and UPFC. A comparison was presented on how the combined operation of FACTS and wind farms can improve system stability and reliability, here social welfare and location marginal pricing were considered for their optimal allocations. In [16], optimal placement of UPFC was determined in the real electrical network by placing it at weak buses determined through PV curves, but no study was done regarding optimal sizing of UPFC. 
PSO was utilized to minimize the objective function of optimal placement and coordination of TCSC and SVC in [17]. The objective was the minimization of the cost associated with the total generation. The method was based on the DC load flow model. Fuzzy logic was used in [18] to determine the optimal locations of UPFC with the objective of enhancing voltage stability here L-index is used to determine weak buses in the system. In [19], mixed integer programming and Taylor series expansion was proposed for the optimal siting of TCSC. A hybrid immune algorithm for the ideal allocation of UPFC to reduce real power losses and to enhance voltage stability is presented in [20]. A hybrid group search optimization technique was utilized for the optimal siting of UPFC to enhance voltage profile and improve ATC in [21]. In [22], the authors proposed PSO and adaptive GSA algorithm for optimum allocation of FACTS for enhancing voltage stability. FACTS devices considered were SVC and UPFC. It is known that PSO converges quickly initially and slows down near-global optimum, hence a hybrid PSO and GA were proposed to reduce power losses and total installation cost for optimum placement of SVC in [23]. The optimal allocation of TCSC with the help of system real power loss sensitivity index was introduced in [24].

Indices which relate variations in loading parameter with regards to reactive power control and variation in loading parameter with regards to the reactance of respective line for optimal siting of SVC and TCSC were introduced in [25]; however, a method for determining optimal coordination and sizing of these FACTS in the network was not discussed. A comparison between the bio-geography based optimizations (BPO), weights-improved PSO (WIPSO) and PSO for optimal allocations of various types of FACTS was presented in [26]. The optimal siting of TCSC, TCVAR, TCPST, and SVC controller in a power system to enhance voltage profile and to reduce real losses using GSA was presented in [27]. In [28], for allocation of the phase shifter, static VAr compensator, and series capacitors line-loss sensitivity-based technique was discussed. A whale optimization algorithm was utilized for the optimal siting, sizing, and coordination of SVC and TCSC in [29]; however, UPFC was not considered in this study. Also, a sensitivity analysis was not carried out for the placement of TCSCs; they were only placed at those lines which are carrying higher reactive power. Thus, these may not be ideal locations for TCSCs. In [30], the ideal siting of UPFC was determined based on the first order reactivity of the transmission line power losses. In [31] sensitivity-based method was used for optimal siting of TCSC and UPFC with the objective to decrease real system power losses and to improve transmission capacity.

None of the work has been carried out in the placement of multiple types of FACTS devices at weak lines and buses determined through sensitivity analysis and simultaneously determining the sizing and coordination of these devices in the power system network. Also, to the best of the authors knowledge whale optimization algorithm has not been tested yet for the optimal sizing and coordination of UPFC in the presence of other FACTS like TCSC and SVC.

In this paper, sensitivity analysis has been carried out initially through the Lmn index and by constructing PV curves of load buses to determine ideal locations for TCSC and SVC. Since the Lmn index is a very good indicator to determine critical lines, hence, the ideal location for TCSC can be determined in the network. Similarly, buses prone to voltage collapse can be easily determined by PV curves. Placing SVC at such buses can highly improve power system stability by providing reactive support and increasing loading capability. Locations of UPFC's are determined by finding out the lines that are carrying higher real power. After placement, sizing, and coordination of these devices are presented through the whale optimization algorithm (WOA), and the results are also compared with PSO and GA. The optimal coordination of FACTS devices is determined with existing reactive power sources in the network, which are tap settings of transformers and reactive power from generators. The objective that is being minimized for this purpose is the total system operational cost, which consists of the cost of active power loss and cost of installation of FACTS. As changing reactive power (VAr) delivered by generators and changing tap settings of transformers are independent of total system operational cost, they are only considered as fitness function variables and are not part of the objective function. The presented methodology is applied to IEEE-30 and 14 bus transmission systems. The rest of paper is arranged as follows. "Section 2" discusses steady-state modeling of SVC, 
TCSC, and UPFC in detail. "Section 3" explains the techniques used for optimal siting of SVC, TCSC and UPFC, "Section 4" discuses mathematical model of the problem. "Section 5" explains WOA in detail and how it is utilized for finding optimal rating and coordination of FACTS. "Section 6" explain the obtained results in detail. Paper is concluded under section "conclusion".

\section{Modeling of FACTS}

SVC is a shunt compensator, and TCSC is a series compensator. UPFCs combine both shunt and series compensation. The shunt compensators can inject or absorb reactive power, and series compensators can control the impedance of transmission lines.

\subsection{Modeling of TCSC}

Depending on the firing angles of thyristors, TCSCs can provide both capacitive or inductive compensations. They are positioned in series with the line and can influence impedance of transmission line. Thus, a TCSC can modify transmission line power carrying capabilities [24]. The mathematical model of TCSC is presented in Figure 2.

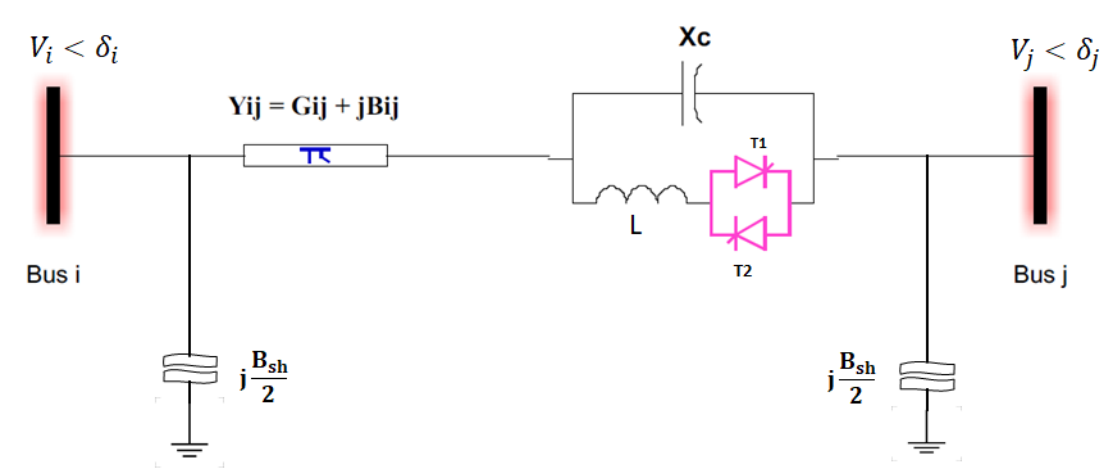

Figure 2. Static model of TCSC.

In the presence of TCSCs, the true and reactive power flow equations from the respective buses can be given as:

$$
\begin{gathered}
P_{i j}=V_{i}^{2} G_{i j}-V_{i} V_{j}\left(G_{i j} \cos \delta_{i j}+B_{i j} \sin \delta_{i j}\right) \\
Q_{i j}=-V_{i}^{2}\left(B_{i j}+B_{s h}\right)-V_{i} V_{j}\left(G_{i j} \sin \delta_{i j}-B_{i j} \cos \delta_{i j}\right) \\
P_{j i}=V_{j}^{2} G_{j i}-V_{j} V_{i}\left(G_{q i} \cos \delta_{j i}-B_{j i} \sin \delta_{j i}\right) \\
Q_{j i}=-V_{j}^{2}\left(B_{i j}+B_{s h}\right)+V_{i} V_{j}\left(G_{i j} \sin \delta_{i j}+B_{i j} \cos \delta_{i j}\right)
\end{gathered}
$$

Here, $V_{i}$ and $V_{j}$ are voltages of sending and receiving end buses, $\delta_{i j}$ is bus angle differences between sending and receiving end, T1 and T2 are thyristors and $B_{s h}$ is the shunt admittance of the line.

$G_{i j}$ and $B_{i j}$ are defined as:

$$
\begin{aligned}
G_{i j} & =\frac{R}{R^{2}+\left(X_{i j}-X_{T c s c}\right)^{2}} \\
B_{i j} & =\frac{-X_{i j}-X_{T c s c}}{R^{2}+\left(X_{i j}-X_{T c s c}\right)^{2}}
\end{aligned}
$$

\subsection{Modeling of $S V C$}

SVCs are parallel connected devices, and they can modify reactive power flows at their point of coupling [32]. Primarily SVCs act as variable inductors or capacitors. Thyristor-switched reactors and 
thyristor-controlled capacitors connected in shunt with the electrical power system. The static model of SVC is shown in Figure 3.

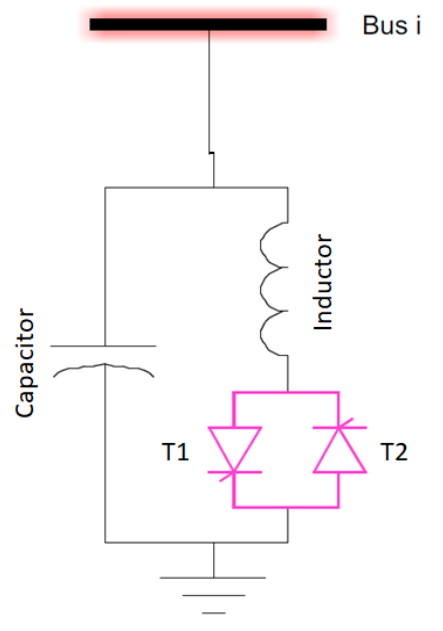

Figure 3. Static model of the SVC.

The relationship between the injected or absorbed VAr at the bus by the SVC is given as follows:

$$
Q_{s v c}=V^{2} B_{s v c}
$$

where Bsvc and ' $\mathrm{V}$ ' are the susceptance and bus voltage, respectively.

\subsection{Modeling of UPFC}

The unified power flows controller (UPFC) was first introduced by Gyugyi in 1992 [33]. They can change all parameters of power systems like angle, impedance, and voltage between the buses, thus modifying power flow in transmission lines [34]. UPFCs are connected in series as well as parallel with line, therefore, they combine both the qualities of series as well as parallel compensators. Although UPFC can control all parameters; however, cost constraints limit their applications. If only voltage compensation is necessary, then the shunt compensator instead of UPFC can be economical, similarly if only controlling line impedances, is required then series compensator instead of UPFC can be a better choice. UPFC has two, three-phase controllable bridges for controlling power flow between the buses [35]. The thyristors firing angle in UPFC ranges from -180 to $+180^{\circ}$. The static-model of UPFC is presented in Figure 4.

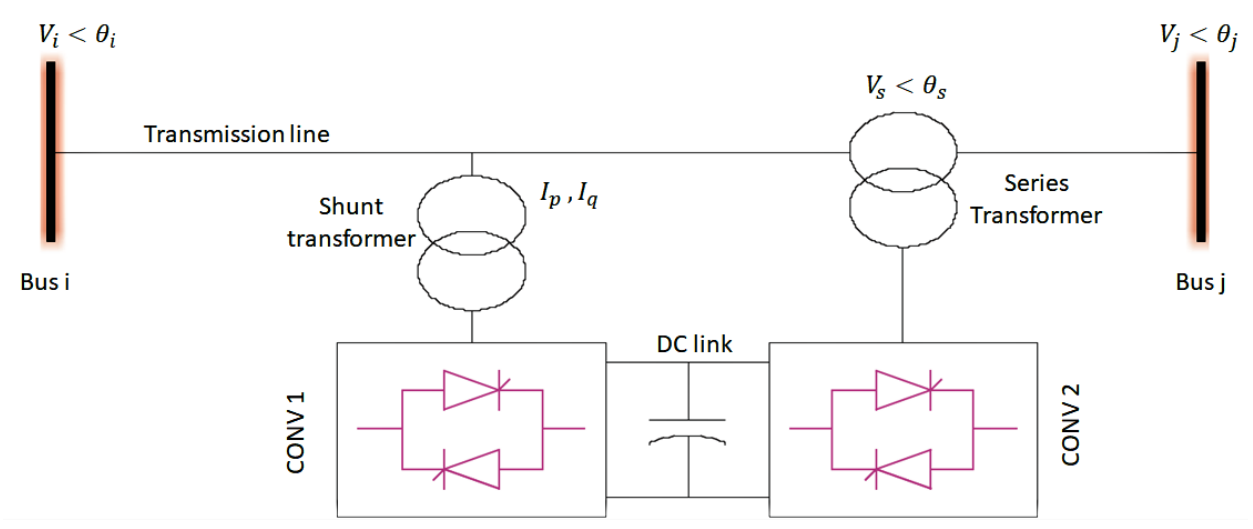

Figure 4. Static model of Unified Power Flow Controller (UPFC). 
Real and reactive power flows between the buses are controlled through series and shunt transformers. CONV 1 and 2 are two, three-phase controllable bridges. The active and reactive power flow equations between the respective buses where UPFC are connected are given as:

$$
\begin{gathered}
P_{i}+V_{s}^{2} G_{i j}+2 V_{i} V_{s} G_{i j} \cos \left(\theta_{s}-\theta_{i}\right)-V_{j} V_{s}\left[G_{i j} \cos \left(\theta_{s}-\theta_{j}\right)-B_{i j} \sin \left(\theta_{s}-\theta_{j}\right)\right]=0 \\
Q_{i}+V_{i} I_{q}-V_{i} V_{s}\left[G_{i j} \sin \left(\theta_{s}-\theta_{i}\right)-B_{i j} \cos \left(\theta_{s}-\theta_{i}\right)\right]=0 \\
P_{j}-V_{j} V_{s}\left[G_{i j} \sin \left(\theta_{s}-\theta_{j}\right)-B_{i j} \cos \left(\theta_{s}-\theta_{j}\right)\right]=0 \\
Q_{j}-V_{j} V_{s}\left[G_{i j} \sin \left(\theta_{s}-\theta_{j}\right)-B_{i j} \cos \left(\theta_{s}-\theta_{j}\right)\right]=0
\end{gathered}
$$

\section{Optimal Placement of FACTS}

The selection of the bus and the line for the placement of FACTS devices largely depends on the topology of the system and desired outcomes. In this work, SVCs are installed to modify the total reactive power flow between the buses. Series compensators TCSCs are used here to change the impedance of the line and, thus, controlling real power flow in the transmission lines. UPFCs are utilized for both voltage improvement and to increase transmission line power flow capacity. FACTS are placed at weak lines and weak buses, the main reason for that is they can modify the overall flow of reactive power in these lines and can indirectly redistribute the power flow to avoid transmission line overloading.

Weak lines and buses are identified using the Lmn Index and by constructing PV curves of all load buses. Although there are a number of ways of shortlisting weak lines and buses in the power system, most of them are either difficult to use or have different drawbacks. For instance, the fast voltage stability index (FVSI) [36] can give accurate results at base case reactive loading; however, as the reactive loading is changed, its results can be inaccurate. Similarly, the voltage collapse proximity index (VCPI) [37] can be utilized to shortlist weak lines and buses in power system; however, it is difficult to implement, plus, Working of VCPI is completely dependent on active power flow through the lines and thus is not a good indicator for different loading scenarios. The Lmn index can give accurate results at all loading points and is easy to implement. Similarly, those buses whose voltages drop rapidly with the increasing load will definitely be weak buses and, thus, are in need of reactive compensations. That is why PV curves can be the best indicators for shortlisting weak buses in power system networks. The TCSCs are placed at weak lines while SVC's are located at weak buses. UPFC location is determined based on the amount of active power carrying condition of the line.

\subsection{Optimal Placement of TCSC}

TCSC's are placed at weak lines which are determined using Lmn index, Lmn index is a very good indicator which shows how much the line is close to instability. The Lines having the Lmn index value close to 1 indicates that the line is critical, for the line to be stable its value is less the 1 . The formula for calculating the Lmn index is shown in Equation (13).

$$
L m n=\frac{4 X Q_{r}}{\left[V_{s} \sin (\theta-\delta)\right]^{2}}
$$

where $X$ is the reactance of the line $Q_{r}$ is the VAr demand at receiving bus, $V_{s}$ is the magnitude of the bus voltage of sending end, $\theta$ is the difference of bus angles, and $\delta$ is the impedance angle. More details about the Lmn index can be found in [38].

\subsection{Optimal Placement of SVC}

Optimal locations of SVC's are determined by analyzing PV curves. PV curves are constructed using the continuation power flow (CPF) technique. Conventional power flow methods fail at bifurcation 
point or maximum load-ability point because of singularity in the Jacobian matrix. Therefore, CPF was developed by Ajjarapu and Christy in 1992 to find power flows at all loading point by slightly modifying the power flow equations. CPF uses predictors and correctors scheme to find solutions for power flow at all loading points as shown in Figure 5.

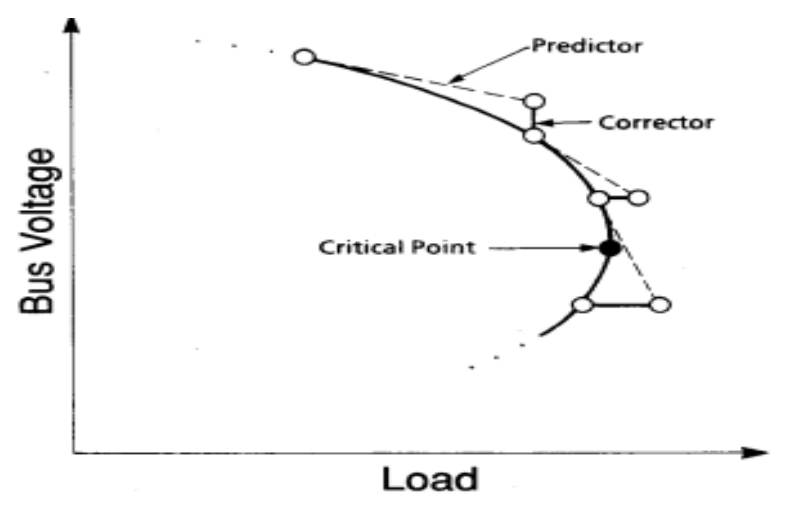

Figure 5. Predictor and corrector scheme used in CPF [39].

Further details about CPF are given in [39]. Since there is no problem with the Jacobian matrix singularity in the CPF technique, the power voltage curve (PV curve) can be obtained for any system at any loading point. PV curves for all load busses are constructed, and buses with higher voltage deviations in response to changing load are considered weak buses and consequently potential locations for SVC placements. CPF is a well-built technique and is available in much commercial software. Here power systems analysis toolbox (PSAT), in MATLAB [40] is used to run CPF and to construct PV curves.

\subsection{Optimal Placement of UPFC}

For optimal placement of the UPFCs those lines which are carrying higher active power are determined, and UPFCs are placed at the starting buses of these lines because their voltage magnitude and corresponding phase angle need to be controlled.

\section{Problem Formulation}

Optimal sizing and coordination of FACTs devices are determined with an objective to minimize operating cost (OC) of the power system, OC consists of active power loss cost, and FACTS device installation cost. For efficient coordination of FACTS devices, tap settings of the transformer and reactive power from generators are also considered as fitness function variables.

The total objective function that needs to be minimized is as follows:

$$
\min \left[C_{P L}+C_{F A C T S}\right]
$$

where

$$
\begin{gathered}
C_{P L}=(\text { Active Power loss }) \times(0.09 \$ / \mathrm{kwh}) \times 365 \times 24 \\
C_{F A C T S}=C_{T C S C}+C_{S V C}+C_{U P F C} \\
C_{T C S C}=0.0015 t^{2}-0.7130 t+153.75\left(\frac{\$}{k V A r}\right) \\
C_{S V C}=0.0003 s^{2}-0.3051 s+127.38\left(\frac{\$}{k V A r}\right) \\
C_{U P F C}=0.0003 u^{2}-0.2961 u+188.22\left(\frac{\$}{k V A r}\right)
\end{gathered}
$$


Cost functions of TCSC, SVC, and UPFC based on Siemens database [11] are given in [41], where $t$, $s$, and $u$ are the sizes of TCSC, SVC, and UPFC in kVAr. Constraints that need to be satisfied are as follows.

1. Bus voltages should be in their appropriate limits as

$$
0.95 \leq V_{j} \leq 1.05
$$

2. Thermal limits of transmission lines

$$
S_{\min } \leq S_{L} \leq S_{\max }
$$

where $S_{L}$ is the apparent power flowing through the line in Mega Volt Ampere (MVA).

3. Generator's reactive power supply limits

$$
Q_{g, \min } \leq Q_{g} \leq Q_{g, \max }
$$

4. Limit on the arrangements of the transformer tap setting

$$
T_{i, \min } \leq T_{i} \leq T_{i, \max }
$$

5. $\quad$ SVC size constraints

$$
-0.9 \leq Z_{S V C} \leq 0.9(p u)
$$

where $\mathrm{Z}_{\mathrm{SVC}}$ is the size of the SVC in pu.

6. TCSC size constraints

$$
-0.8 X_{L} \leq X_{T C S C} \leq 0.2 X_{L} p u
$$

where $X_{T C S C}$ is the size of the TCSC in pu

\section{Whale Optimization Algorithm}

The whale optimization algorithm was first presented by Lewis and Mirjalil in 2016 [42]. It's a meta-heuristic optimization technique. As our optimization problem is highly non-linear because in every iteration power loss is calculated, metaheuristic optimization techniques are suitable solving techniques. WOA is stimulated by Humpback-whale special hunting technique called the bubble-net feeding method. A group of humpback-whales encircle the prey in a specific pattern; initially, they create sound and dive deep to push small krill's and fishes to the surface while releasing bubbles in circles to make a trap, after this, all whales come up with mouth open and hunt down their prey. The optimization algorithm inspired by this special hunting technique can be mathematically modeled by following three main steps.

1. Encircling prey

2. Exploitation phase

3. Exploration phase

Details of each step and their respective modeling are discussed below.

\subsection{Encircling Prey}

Humpback-whales encircles the prey because they know the location of prey. The position of each humpback whales or search agents is updated according to the current optimal candidate solution. This prey encircling behavior can be expressed mathematically as:

$$
D=\left|C \times X_{B(t)}-X_{t}\right|
$$




$$
X(t+1)=X_{B(t)}-A \times D
$$

where $X_{B(t)}$ represent position-vector of best-solution at each iteration, | | represent absolute value $X_{t}$ represent the location of the search agent, and vectors ' $\mathrm{C}$ ' and ' $\mathrm{A}$ ' are defined as.।:

$$
\begin{gathered}
A=2 a \times r_{1}-a \\
C=2 \times r_{2}
\end{gathered}
$$

As iterations proceed, ' $a$ ' is linearly decreased from $[2,0]$, whereas values of $r_{2}$ and $r_{1}$ range from $[0,1]$.

\subsection{Exploitation Phase}

Humpback-whales hunt their prey by bubble net mechanism. In this technique bubbles are released around the prey to make a trap then Humpback-whale move around the prey in the shrinking circle as well as come up and update its position in the spiral shape. This strategy can be mathematically modeled as follows.

\subsubsection{Shrinking-Encircling Technique}

To achieve this, the value of ' $a$ ' in Equation (28) is decreased from 2 to 0 as the iteration proceeds. Thus ' $A$ ' will have a random value from $[-a, a]$. From Figure 6 , it can be seen that different positions are available by moving from $\left(\mathrm{X}^{*}, \mathrm{Y}^{*}\right)$ to $(\mathrm{X}, \mathrm{Y})$ by changing values of $\mathrm{A}$.

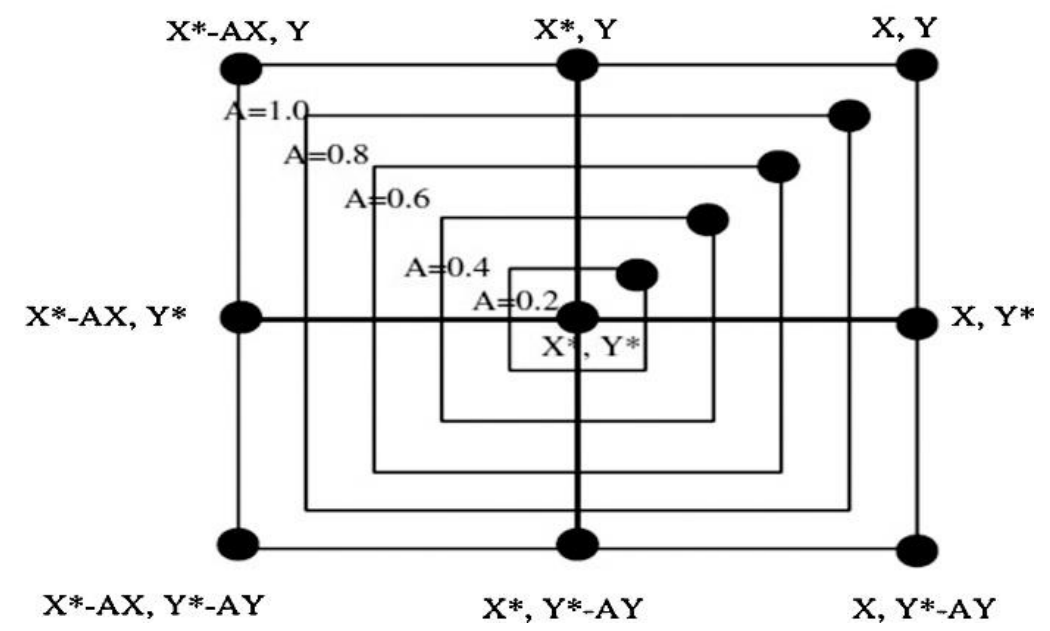

Figure 6. Shrinking-encircling technique [42].

\subsubsection{Spirals-Updating Position Technique}

Here the total distance from prey to humpback-whales is determined, and a helix-shape movement equation for search agent is created, which can be written as follows.

$$
X_{(t+1)}=D^{*} \times e^{b t} \times \cos (2 \pi l) \times X_{B(t)}
$$

where ' 1 ' ranges from $[-1,1],{ }^{\prime} b$ ' is a constant and ' $D$ ' represent distance between $j$ th whale and the prey (best-solution), $D=\left|X_{B(t)}-X_{t}\right|$.

There is a $50 \%$ probability that the Humpback-whale will follow either shrinking circular or a helix-shaped movement as it moves around its prey. Thus, its total swim around the prey during its hunt down can be mathematically modeled as: 


$$
X_{(t+1)}=\left\{\begin{array}{c}
X_{B(t)}-A \times D \text { if } p<0.5 \\
D^{*} \times e^{b t} \times \cos (2 \pi l) \times X_{B(t)} \text { if } p \geq 0.5
\end{array}\right.
$$

' $p$ ' represents a random value ranging from $[0,1]$. Visual representation of how the position will be updated from Equation (31) is shown in Figure 7.

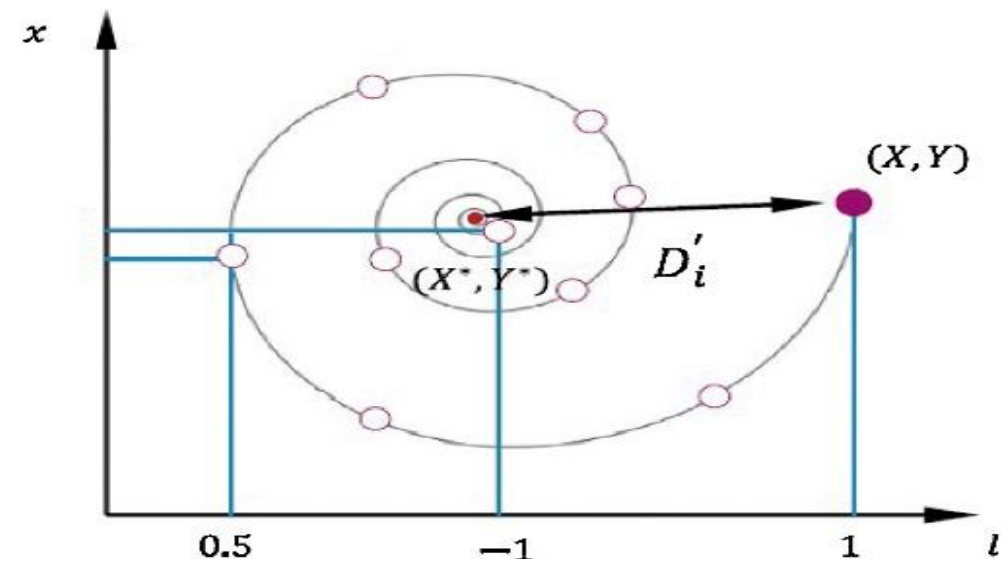

Figure 7. Spiral-updating position technique [42].

\subsection{Searching for Prey (Exploration-Phase)}

In order to provide a global search, the search-agent or Humpback-whale explores the best-solution and updates its position based on another randomly selected search-agent. This behavior can be mathematically expressed as follows:

$$
\begin{gathered}
D=\left|C X_{r}-X\right| \\
X(t+1)=X_{r}-A \times D
\end{gathered}
$$

This makes WOA a global search optimization algorithm. Where $X_{r}$ is the position vector of randomly selected search agent/whale.

Summarizing the above method, the WOA starts with a random particle having their respective fitness function values. As the iteration proceeds, each humpback-whale/search-agent upgrades its position in two different ways, either with regards to randomly selected particle or overall best particle present so far. A random particle is chosen when the magnitude of $A>1$, while the best particle/solution is selected when the magnitude of $\mathrm{A}<1$. To switch between different phases (exploration or exploitation), values of parameter ' $a$ ' is varied, which ranges from $[2,0]$. Finally, when termination criteria are met WOA comes to an end.

To find optimal sizes of multiple FACTS devices (SVC, TCSC, and UPFC) as well as settings of power system components (transformers and generators) by the WOA, fixing dimensions of search-agent/whale is a major decision. In our case, there are a total of 18 decision variables for both IEEE 14 and 30 bus systems, as shown in Table 2. Therefore, in our implementation of WOA,

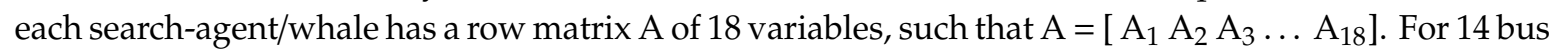
systems, $\mathrm{A}_{1-4}$ decision variables are TCSC sizes, $\mathrm{A}_{5-8}$ are SVC sizes, $\mathrm{A}_{9-11}$ are UPFC sizes, $\mathrm{A}_{12-14}$ are tap setting values of the transformers, and $\mathrm{A}_{15-18}$ are the reactive power values of generators. Similarly, each search agent/whale is composed and initialized for the 30 bus system. The initial population matrix of 100 search-agents/whales is stored in a column matrix of $100 \times 18$ variables. As WOA proceeds, exploration and exploitation phases of WOA optimize values of the decision variables.

Figure 8 presents a flowchart of proposed WOA for optimal sizing and coordination of FACTS devices. 


\subsection{Flowchart}

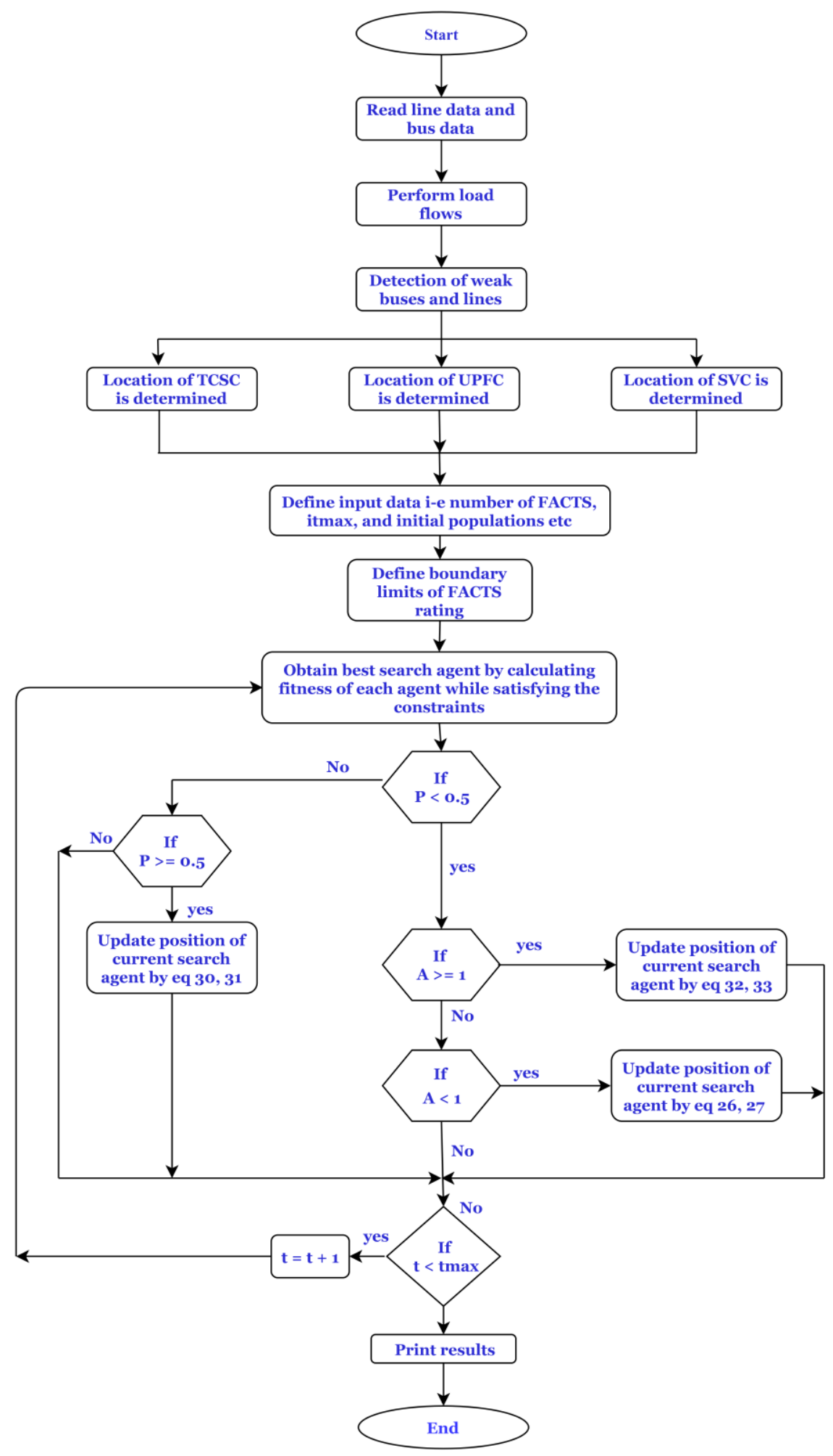

Figure 8. Flow chart of the proposed WOA.

\section{Results and Discussions}

The proposed method of optimal siting, sizing and coordination of FACTS devices is applied on IEEE-14 and the 30 bus systems. The results and discussions of both the test systems are given below. 


\subsection{Test Case IEEE-14 Bus System}

The IEEE-14 bus system [43] consists of five power generating units, twenty transmission lines, and the total of nine load buses. From the load flows, the total reactive and real power losses before placement of FACTS devices are 13.393 MW and 54.54 MVAr. The total real power demand is $259 \mathrm{MW}$, and the total real power generation capacity is $772.4 \mathrm{MW}$. The total reactive power demand and generation are 73.5 MVAr and 82.4 MVAr, respectively. The optimal locations for TCSCs using the Lmn index are branches $8,9,15$, and 18. By constructing the PV curves of all load buses in PSAT the buses with higher voltage deviations are $7,9,11$, and 14 , so they are considered weak buses and the SVCs are positioned at these locations. In the IEEE-14 bus system, lines 1,3, and 10 carry higher active power; these lines are connected between buses $1-2,2-3$, and 5-6, where buses 2,3 , and 5 are starting buses and their voltage magnitude and angle have to be controlled. Consequently, UPFCs are connected at buses 2, 3, and 5. The one-line diagram of the IEEE 14 bus system and visual representation of the different FACTS on it are shown in Figure 9. Table 2 presents the total number of variables used in the optimization algorithms.

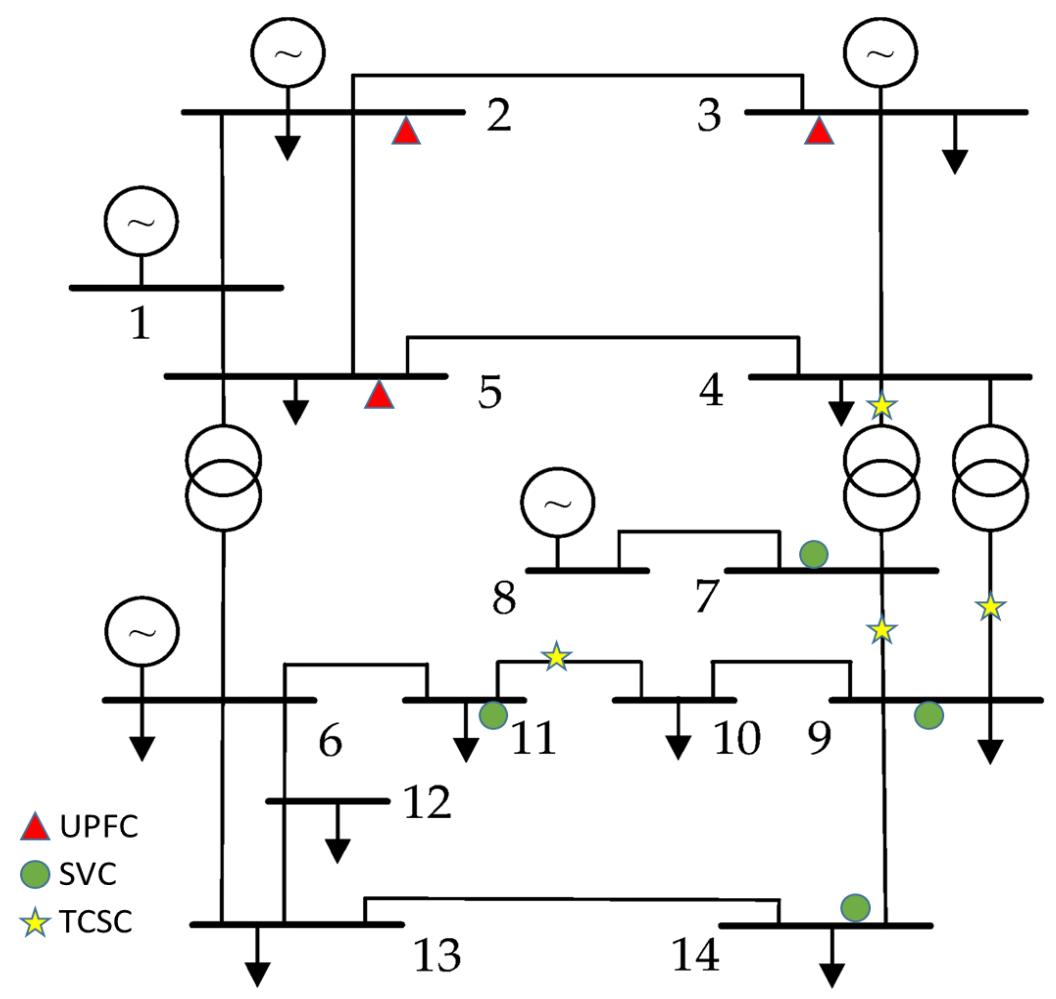

Figure 9. FACTS devices placement on IEEE-14 bus system.

Table 2. Number of variables for the test systems in the presence of the FACTS devices.

\begin{tabular}{cccccc}
\hline Test-System & $\begin{array}{c}\text { No. of } \\
\text { TCSC }\end{array}$ & $\begin{array}{c}\text { No. of } \\
\text { SVCs }\end{array}$ & $\begin{array}{c}\text { No. of } \\
\text { UPFCs }\end{array}$ & $\begin{array}{c}\text { No. of Tap Settings } \\
\text { of Transformer }\end{array}$ & $\begin{array}{c}\text { Var Generation } \\
\text { Units }\end{array}$ \\
\hline IEEE-14 bus system & 4 & 4 & 3 & 3 & 4 \\
IEEE-30 bus system & 3 & 3 & 3 & 4 & 5 \\
\hline
\end{tabular}

After defining FACTs positions using respective strategies, TCSCs, SVCs, and UPFCs are placed at their optimal locations, and different optimization algorithms like WOA, PSO, and GA are utilized to minimize the objective function. For WOA, the total of 100 whales or search agents are considered, and the iterations are run for 100 times. For IEEE-14 bus system total of 18 fitness function variables are considered here. The variables comprise of a total number of FACTS devices, total transformer taps settings, and reactive power generation units. Fitness function variables are nothing but just a 
string of variables carrying different ratings or sizes, which affect the fitness function value. The size of variables is changed in every iteration and optimized by an algorithm.

Figure 10 presents a comparison of reduction in true power losses using different techniques at various loading scenarios before and after the placement of the FACTS devices.

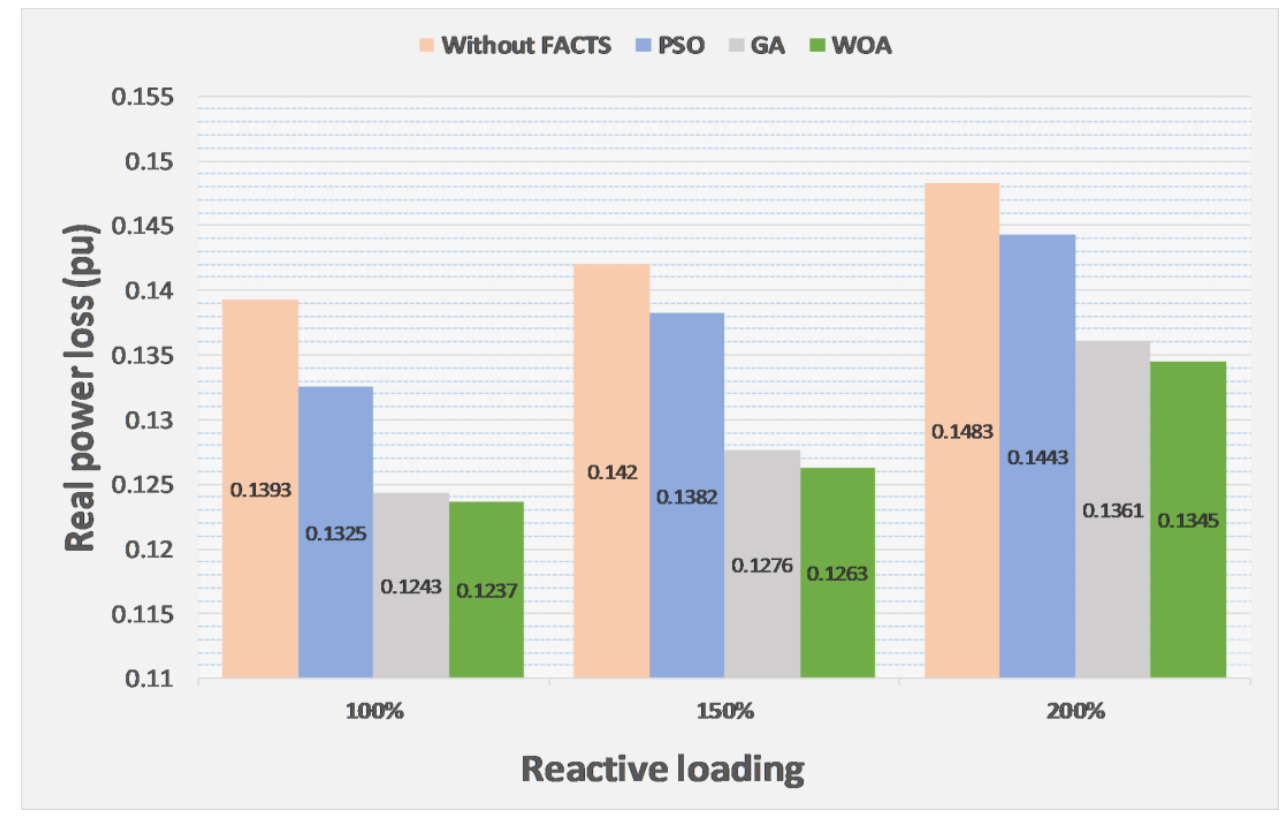

Figure 10. Comparison of real losses without and with FACTS in th IEEE-14 system using different.

Table 3 shows the comparison of the operating-cost (OC) in the IEEE-14 system with and without FACTS using different techniques. Without FACTS devices, the OC only consists of cost due to active power loss. In this work, energy cost is considered as $0.092 \$ / \mathrm{kwh}$. PSO is run for 100 iterations with 100 particles. Similarly, WOA is run for 100 times, and the number of whales/search-agent taken are 100, GA is implemented in the MATLAB optimtool. After positioning of the FACTS devices, the total OC consists of the cost of the true power loss and the cost of installation of the FACTs. Variables here are the total number of tap settings of transformers, VAr generation units, and the total FACTS devices installed. Table 4 presents the optimal values of these variables using different techniques at different reactive power loadings.

From Table 3 net savings can be calculated as (A-B). A comparison of net saving for a whole year using PSO, GA, and WOA at different reactive loading is presented in Figure 11.

Table 3. Cost of power loss and operating cost of the system with FACTS in the IEEE-14 bus system using different techniques.

\begin{tabular}{ccccc}
\hline $\begin{array}{c}\text { Percentage } \\
\text { Reactive } \\
\text { Loadings }\end{array}$ & $\begin{array}{c}\text { Total Cost of Power } \\
\text { Loss of System (A) } \\
\mathbf{\$}\end{array}$ & $\begin{array}{c}\text { Algorithm Used to } \\
\text { Minimize Objective } \\
\text { Function }\end{array}$ & $\begin{array}{c}\text { FACTS Devices } \\
\text { Cost } \\
\mathbf{( \$ )}\end{array}$ & $\begin{array}{c}\text { Operating Cost with } \\
\text { FACTS Devices (B) } \\
\mathbf{\$}\end{array}$ \\
\hline \multirow{2}{*}{200} & \multirow{2}{*}{$1.1952 \times 10^{7}$} & PSO & $3.433 \times 10^{5}$ & $1.172 \times 10^{7}$ \\
& & GA & $2.988 \times 10^{5}$ & $1.1029 \times 10^{7}$ \\
& \multirow{2}{*}{$1.1442 \times 10^{7}$} & WOA & $2.870 \times 10^{5}$ & $1.0891 \times 10^{7}$ \\
\hline \multirow{2}{*}{150} & & PSO & $3.033 \times 10^{5}$ & $1.1199 \times 10^{7}$ \\
& \multirow{2}{*}{$1.1226 \times 10^{7}$} & GA & $2.580 \times 10^{5}$ & $1.0318 \times 10^{7}$ \\
& & WOA & $2.525 \times 10^{5}$ & $1.021 \times 10^{7}$ \\
\hline \multirow{2}{*}{100} & PSO & $3.677 \times 10^{5}$ & $1.0714 \times 10^{7}$ \\
& & GA & $3.202 \times 10^{5}$ & $1.012 \times 10^{7}$ \\
\end{tabular}




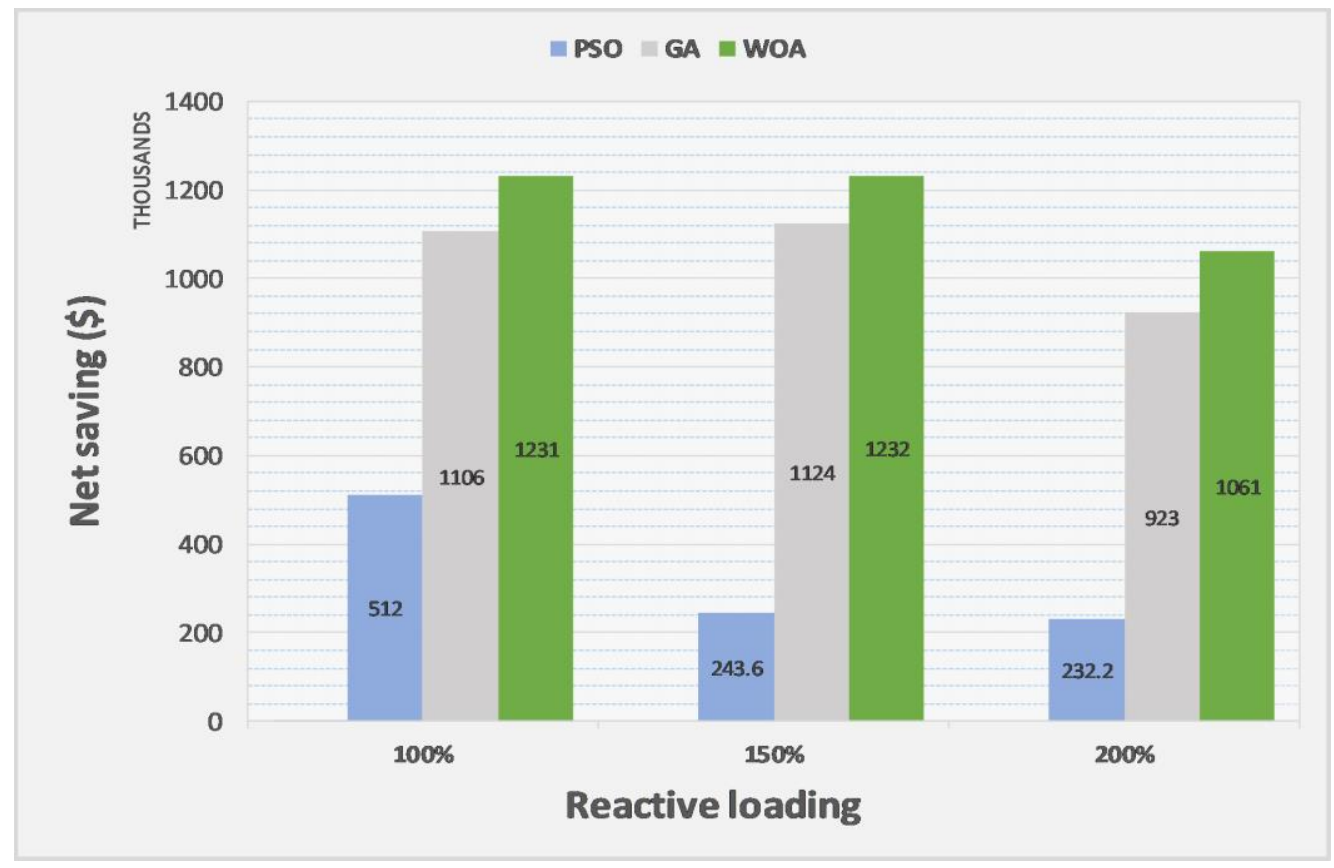

Figure 11. Comparison of the net savings at various reactive loading.

Table 4. Optimal setting of variables by PSO, GA, and WOA at various loading scenarios in IEEE-14 bus system.

\begin{tabular}{cccccccccc}
\hline $\begin{array}{c}\text { Fitness Function } \\
\text { Variables }\end{array}$ & $\begin{array}{c}\text { Optimal Settings at 200\% } \\
\text { Reactive Loading } \\
\text { (p.u) }\end{array}$ & $\begin{array}{c}\text { Optimal Settings at 150\% } \\
\text { Reactive Loading } \\
\text { (p.u) }\end{array}$ & $\begin{array}{c}\text { Optimal Settings at 100\% } \\
\text { Reactive Loading } \\
\text { (p.u) }\end{array}$ \\
\hline SVC (7) & PSO & GA & WOA & PSO & GA & WOA & PSO & GA & WOA \\
SVC (9) & 0.003 & 0.201 & 0.041 & 0.051 & 0.160 & 0.029 & 0.011 & 0.090 & 0.001 \\
SVC (11) & 0.022 & 0.170 & 0.034 & 0.012 & 0.120 & 0.290 & 0.061 & 0.052 & 0.001 \\
SVC (14) & 0.194 & 0.112 & 0.005 & 0.125 & 0.070 & 0.094 & 0.002 & 0.040 & 0.061 \\
TCSC (8) & 0.127 & 0.061 & 0.002 & 0.102 & 0.102 & 0.081 & 0.194 & 0.150 & 0.001 \\
TCSC (9) & 0.002 & 0.014 & 0.050 & 0.086 & 0.124 & 0.005 & 0.034 & 0.102 & 0.060 \\
TCSC (15) & 0.014 & 0.024 & 0.050 & 0.018 & 0.001 & 0.059 & 0.011 & 0.016 & -0.040 \\
TCSC (18) & 0.001 & 0.000 & 0.019 & 0.001 & 0.017 & 0.000 & 0.001 & 0.011 & 0.091 \\
UPFC (3) & 0.006 & 0.019 & 0.004 & 0.000 & 0.008 & 0.031 & 0.170 & 0.001 & 0.001 \\
UPFC (2) & 0.280 & 0.000 & 0.001 & 0.002 & 0.009 & 0.001 & 0.003 & 0.039 & 0.100 \\
UPFC (5) & 0.001 & 0.001 & 0.009 & 0.070 & 0.001 & 0.311 & 0.000 & 0.002 & 0.000 \\
QG (2) & 0.122 & 0.154 & 0.601 & 0.015 & 0.681 & 0.600 & 0.312 & 0.613 & 0.551 \\
QG (3) & 0.321 & 0.277 & 0.581 & 0.419 & 0.083 & 0.369 & 0.389 & 0.667 & 0.481 \\
QG (6) & 0.415 & 0.091 & 0.182 & 0.225 & 0.184 & 0.487 & 0.413 & 0.519 & 0.101 \\
QG (8) & 0.087 & 0.623 & 0.163 & 0.451 & 0.212 & 0.098 & 0.513 & 0.082 & 0.682 \\
TAP (8) & 0.982 & 1.018 & 0.900 & 0.913 & 0.976 & 1.0300 & 0.905 & 1.054 & 0.901 \\
TAP (9) & 0.995 & 0.919 & 0.991 & 0.985 & 0.992 & 0.902 & 0.991 & 0.981 & 0.978 \\
TAP (10) & 0.957 & 0.996 & 0.985 & 0.996 & 1.000 & 1.013 & 0.916 & 0.985 & 0.901 \\
\hline
\end{tabular}

Figures 12-14 show the variation of operation-cost by adding FACTS devices to the network using different techniques. 


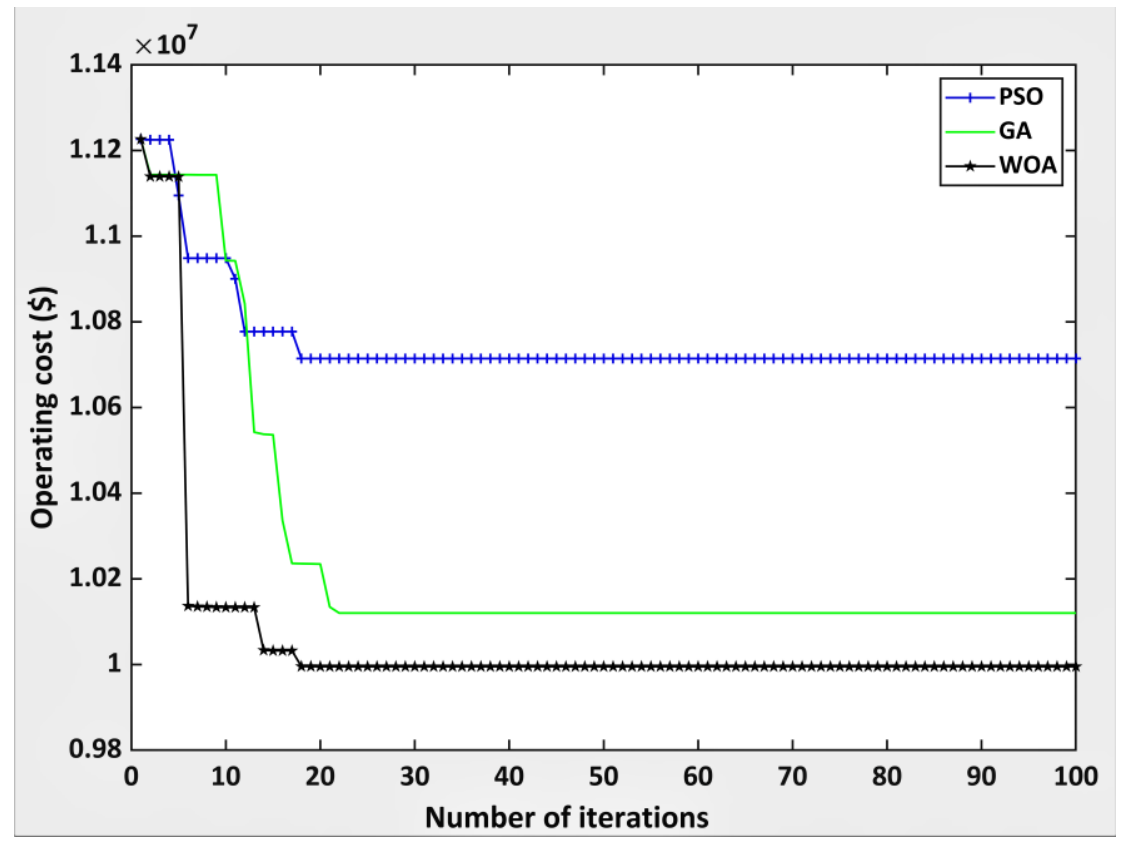

Figure 12. Change in operating cost with the addition of FACTS at base-case using PSO, GA, and WOA.

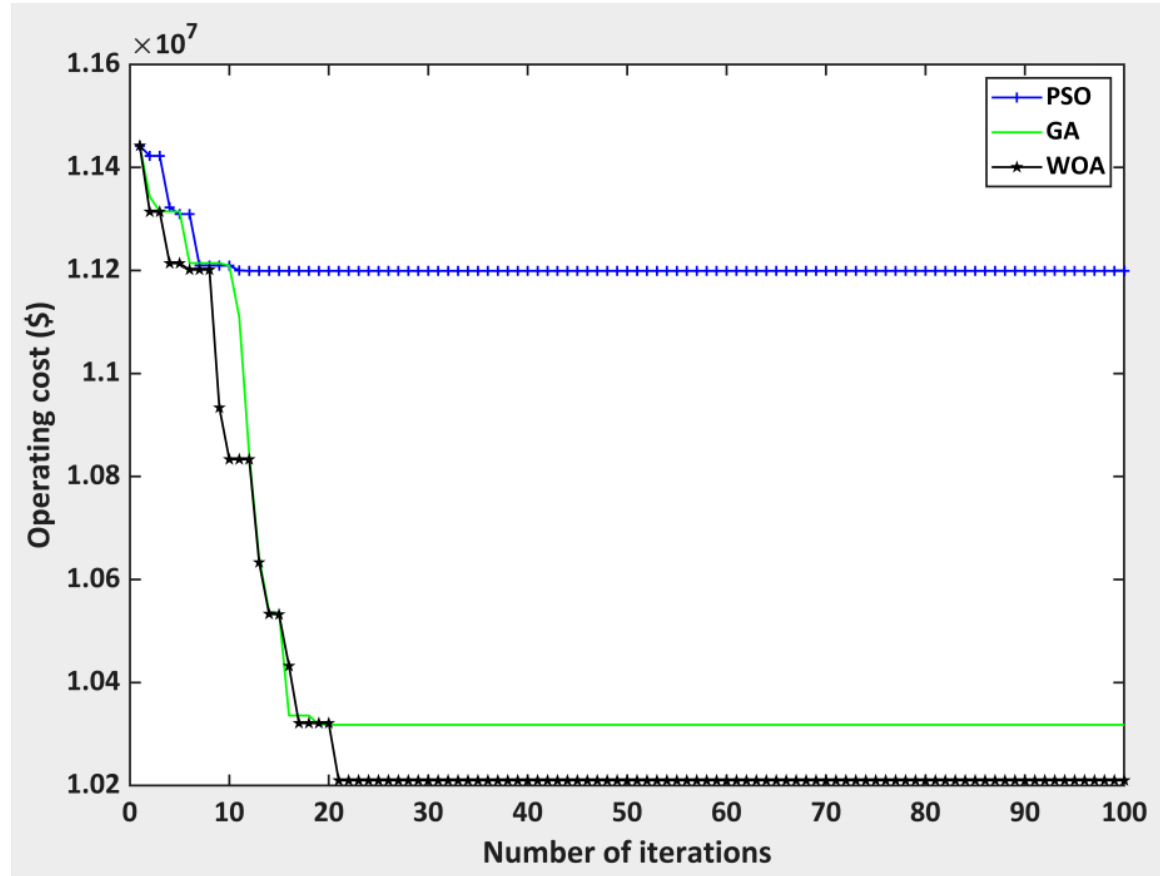

Figure 13. Change in Operating cost with the addition of FACTS at $150 \%$ reactive loading using PSO, GA, and WOA. 


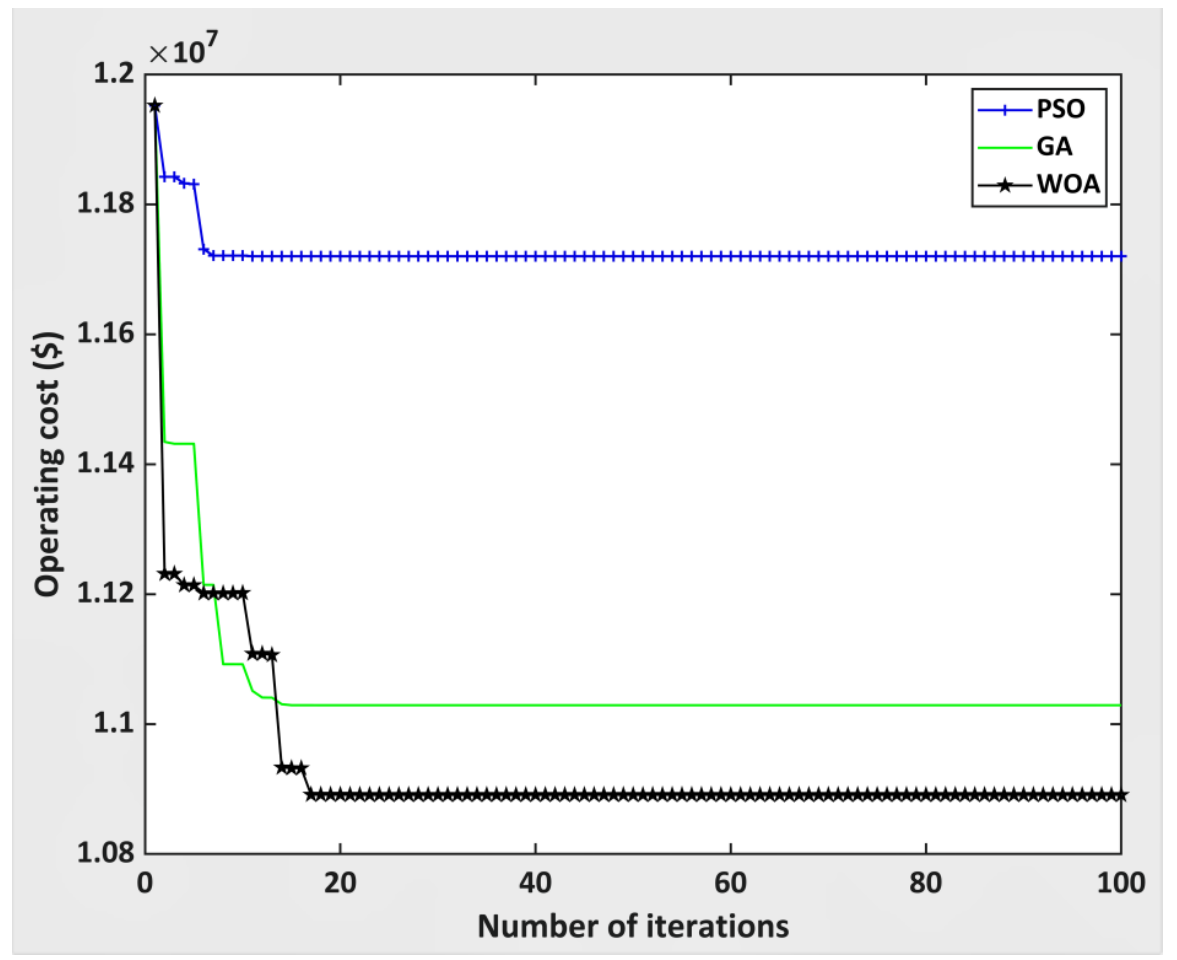

Figure 14. Change in operating cost with the addition of FACTS at $200 \%$ reactive loading using PSO, GA, and WOA.

From Figure 11, we can see that at the base case reactive loading, WOA converged at $0.9995 \times 10^{7}(\$)$ as compared to PSO and GA, which converged at $1.0714 \times 10^{7}(\$)$ and $1.012 \times 10^{7}(\$)$, respectively. Hence WOA saved $719 \times 10^{3}(\$)$ more as compared to PSO and $125 \times 10^{3}(\$)$ more as compared to GA. Similarly, for all other loading scenarios. Hence WOA has better convergence characteristics and provided more savings in operating cost as compared to PSO and GA.

The better performance of WOA is because of its highly effective exploration and exploitation. Equation (33) provides exploration ability to WOA, whereas Equations (30) and (31) control convergence and exploitation of WOA, respectively. Moreover, an adaptive A (Equation (28)) allows WOA to switch between exploration and exploitation. These advantages of WOA enable it to avoid the local optima and converge at global optimum solution highly. WOA is considered to be a global optimizer as compared to PSO and GA, which do not guarantee global optima and are considered as a local optimizer. Also, PSO slows down near global optima because the position of particles is mainly dependent on its personal best $\left(\mathrm{P}_{\text {best }}\right)$ and global best $\left(\mathrm{G}_{\text {best }}\right)$, thus, increasing its chances of jamming at some local optima instead of the global optima [44].

\subsection{Test Case IEEE 30 Bus System}

IEEE-30 bus system [43] consists of 41 transmission lines and six generators. Total real power generation capacity is $900.2 \mathrm{MW}$, total connected load is $283.4 \mathrm{MW}$ and reactive load demand is 126.2 MVAr. Total true and reactive power losses are 17.56 MW and 67.69 MVAr with an energy cost of $0.092 \$ / \mathrm{kWh}$ total initial operating cost without FACTs devices at a base case loading of $1.4152 \times 10^{7} \$$. Using the Lmn sensitivity index, suitable locations for the TCSCs placement in the IEEE 30 bus system are branches 7, 15, and 20. Similarly, using the PV curves, buses with higher voltage deviations with increasing loads are 26,29, and 30, thus, they are weak buses and the SVC's are positioned at these buses.

In IEEE-30 bus system lines 4, 7, 9 are carrying higher active power and are connected between buses 3-4, 4-6 and 6-7 thus UPFCs are connected at buses 3, 4, and 6 to control their voltage magnitude and respective angles. After connecting each FACTS device at their optimal locations WOA, PSO, 
and GA are utilized to determine optimal ratings of FACTS devices that minimize the objective function. The locations of the FACTS devices and one-line diagram of the IEEE-30 bus system are shown in Figure 15.

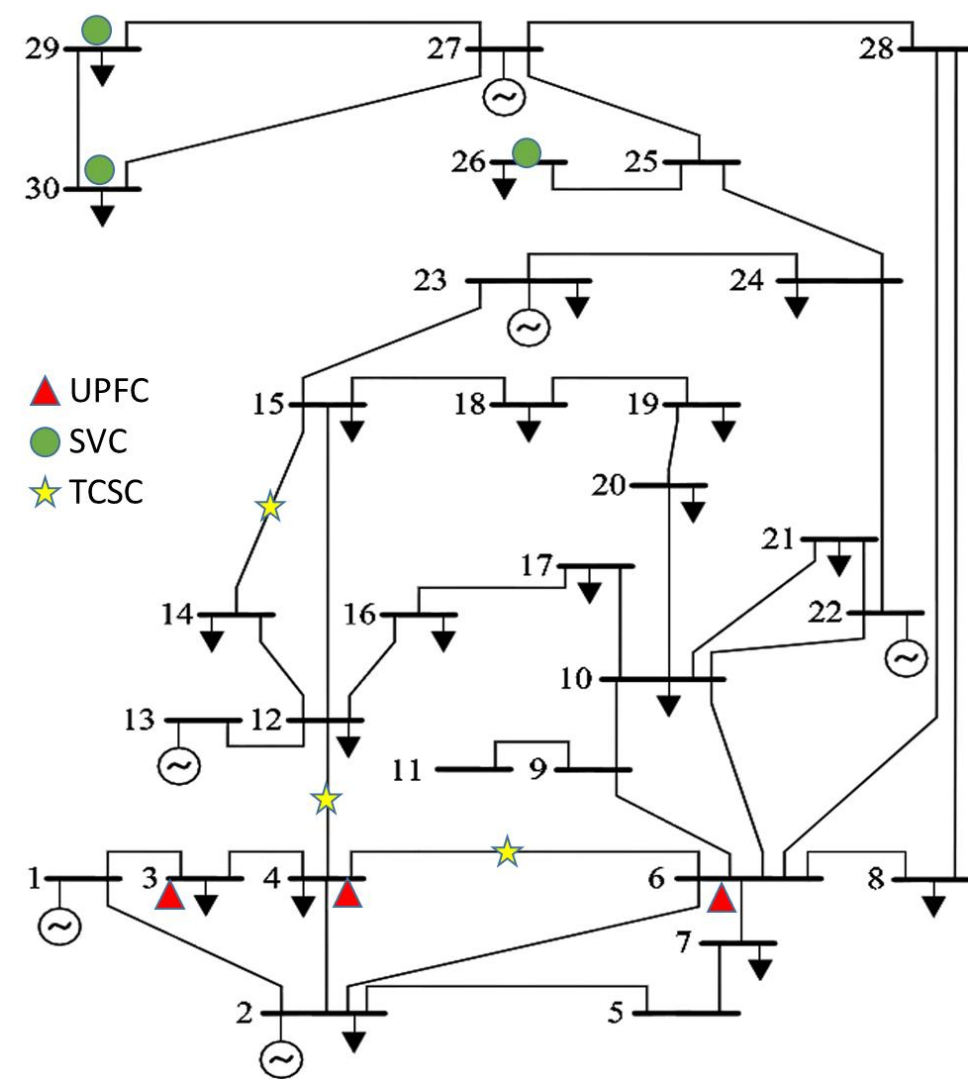

Figure 15. FACTS devices placement on the IEEE-30 bus system.

Total true power losses before and after placing FACTS at different reactive loadings are presented in Figure 16.

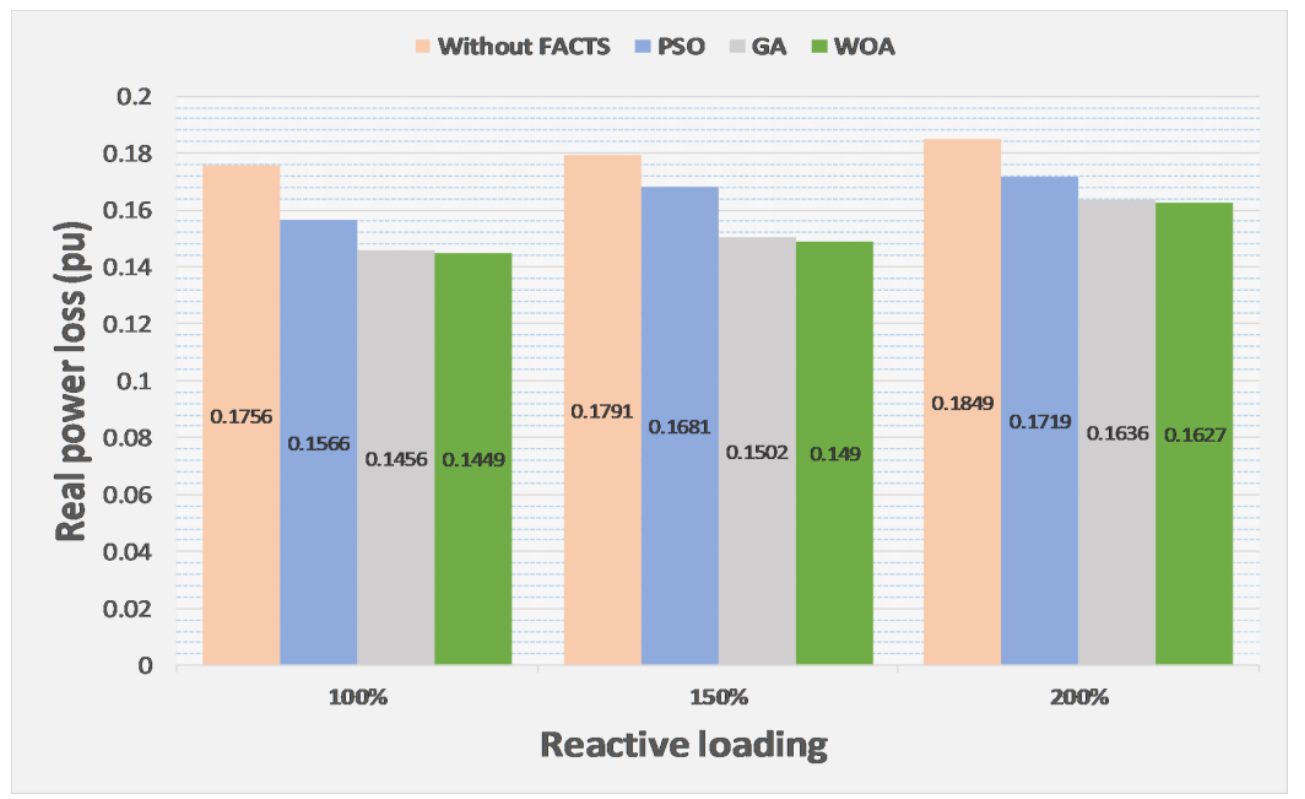

Figure 16. Comparison of real losses without and with FACTS in the IEEE-30 system using different techniques. 
Table 5 shows a comparison of the OC in the IEEE-30 bus system using different techniques, OC without FACTS is just the cost of active power loss. After placing FACTS, the OC consists of real power loss costs and FACTS installation costs.

Table 5. Cost of power loss and operating cost of the system with the FACTS in the IEEE-30 bus system using different techniques.

\begin{tabular}{ccccc}
\hline $\begin{array}{c}\text { Percentage } \\
\text { Reactive Loading }\end{array}$ & $\begin{array}{c}\text { Total Cost of } \\
\text { Power Loss of } \\
\text { System (A) (\$) }\end{array}$ & $\begin{array}{c}\text { Algorithm Used to } \\
\text { Minimize Objective } \\
\text { Function }\end{array}$ & $\begin{array}{c}\text { FACTS Devices } \\
\text { Cost (\$) }\end{array}$ & $\begin{array}{c}\text { Operating Cost } \\
\text { with FACTS (B) (\$) }\end{array}$ \\
\hline \multirow{2}{*}{200} & \multirow{2}{*}{$1.4902 \times 10^{7}$} & PSO & $3.774 \times 10^{5}$ & $1.393 \times 10^{7}$ \\
& \multirow{2}{*}{$1.443 \times 10^{7}$} & GA & $3.517 \times 10^{5}$ & $1.325 \times 10^{7}$ \\
& & WOA & $3.527 \times 10^{5}$ & $1.318 \times 10^{7}$ \\
\hline \multirow{2}{*}{150} & PSO & $3.479 \times 10^{5}$ & $1.3601 \times 10^{7}$ \\
& \multirow{2}{*}{$1.4152 \times 10^{7}$} & GA & $3.082 \times 10^{5}$ & $1.215 \times 10^{7}$ \\
& & WOA & $3.028 \times 10^{5}$ & $1.205 \times 10^{7}$ \\
\hline \multirow{2}{*}{100} & GSO & $3.136 \times 10^{5}$ & $1.266 \times 10^{7}$ \\
& & WOA & $2.908 \times 10^{5}$ & $1.177 \times 10^{7}$ \\
\end{tabular}

Comparison of the net savings (A-B) for a whole year with FACTS devices sized and coordinated using different techniques are shown in Figure 17.

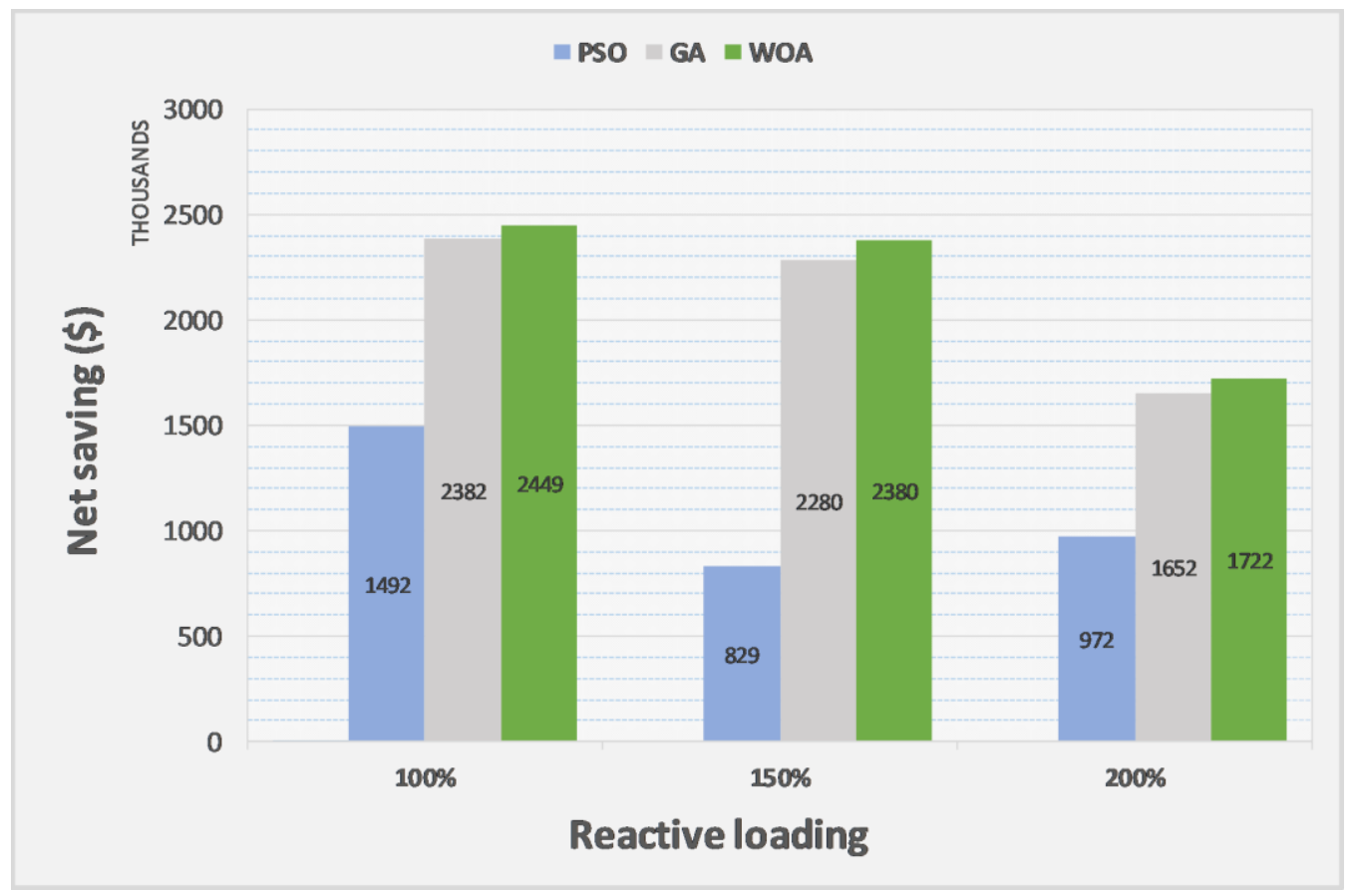

Figure 17. Comparison of the net savings at various reactive loadings using different techniques.

Optimal variables setting at different reactive loading using PSO, GA and WOA is presented in Table 6. 
Table 6. Optimal settings of variables by PSO, GA, and WOA at various loading scenarios in IEEE-30 bus system.

\begin{tabular}{cccccccccc}
\hline $\begin{array}{c}\text { Fitness Function } \\
\text { Variables }\end{array}$ & \multicolumn{3}{c}{$\begin{array}{c}\text { Optimal Setting at 200\% } \\
\text { Reactive Loading }\end{array}$} & \multicolumn{3}{c}{$\begin{array}{c}\text { Optimal Setting at 150\% } \\
\text { Reactive Loading }\end{array}$} & \multicolumn{2}{c}{$\begin{array}{c}\text { Optimal Setting at 100\% } \\
\text { Reactive Loading }\end{array}$} \\
\hline & PSO & GA & WOA & PSO & GA & WOA & PSO & GA & WOA \\
SVC (26) & 0.041 & 0.045 & 0.057 & 0.051 & 0.031 & 0.049 & 0.021 & 0.032 & 0.000 \\
SVC (29) & 0.194 & 0.211 & 0.049 & 0.143 & 0.117 & 0.240 & 0.078 & 0.046 & 0.010 \\
SVC (30) & 0.142 & 0.108 & 0.0418 & 0.072 & 0.093 & 0.140 & 0.055 & 0.048 & 0.018 \\
TCSC (15) & 0.026 & 0.014 & 0.050 & 0.086 & 0.124 & 0.005 & 0.034 & 0.102 & 0.060 \\
TCSC (7) & 0.015 & 0.024 & 0.061 & 0.000 & 0.067 & 0.073 & 0.040 & 0.043 & 0.051 \\
TCSC (20) & 0.048 & 0.008 & 0.050 & 0.018 & 0.001 & 0.059 & 0.011 & 0.016 & -0.040 \\
UPFC (3) & 0.018 & 0.011 & 0.241 & 0.011 & 0.028 & 0.051 & 0.017 & 0.011 & 0.041 \\
UPFC (6) & 0.003 & 0.034 & 0.004 & 0.037 & 0.005 & 0.031 & 0.023 & 0.013 & 0.041 \\
UPFC (4) & 0.025 & 0.028 & 0.031 & 0.024 & 0.009 & 0.051 & 0.016 & 0.037 & 0.001 \\
QG (2) & 0.318 & 0.158 & 0.590 & 0.267 & 0.042 & 0.131 & 0.198 & 0.267 & 0.610 \\
QG (5) & 0.248 & 0.103 & 0.290 & 0.364 & 0.698 & 0.591 & 0.014 & 0.388 & 0.509 \\
QG (8) & 0.023 & 0.191 & 0.501 & 0.611 & 0.045 & 0.610 & 0.581 & 0.314 & 0.098 \\
QG (11) & 0.518 & 0.208 & 0.191 & 0.317 & 0.142 & 0.519 & 0.318 & 0.301 & 0.611 \\
QG (13) & 0.032 & 0.605 & 0.189 & 0.345 & 0.298 & 0.181 & 0.676 & 0.097 & 0.184 \\
TAP (11) & 0.914 & 0.904 & 1.034 & 0.982 & 0.914 & 0.991 & 0.948 & 0.903 & 1.032 \\
TAP (12) & 1.038 & 0.981 & 1.050 & 0.938 & 0.901 & 0.990 & 0.934 & 1.012 & 0.920 \\
TAP (15) & 0.902 & 0.958 & 1.043 & 0.984 & 0.918 & 1.020 & 0.931 & 0.907 & 1.018 \\
TAP (36) & 0.918 & 0.905 & 0.988 & 0.901 & 0.926 & 1.038 & 0.938 & 0.994 & 0.991 \\
\hline
\end{tabular}

Figures 18-20 present variation of operating cost at different reactive loading using different techniques.

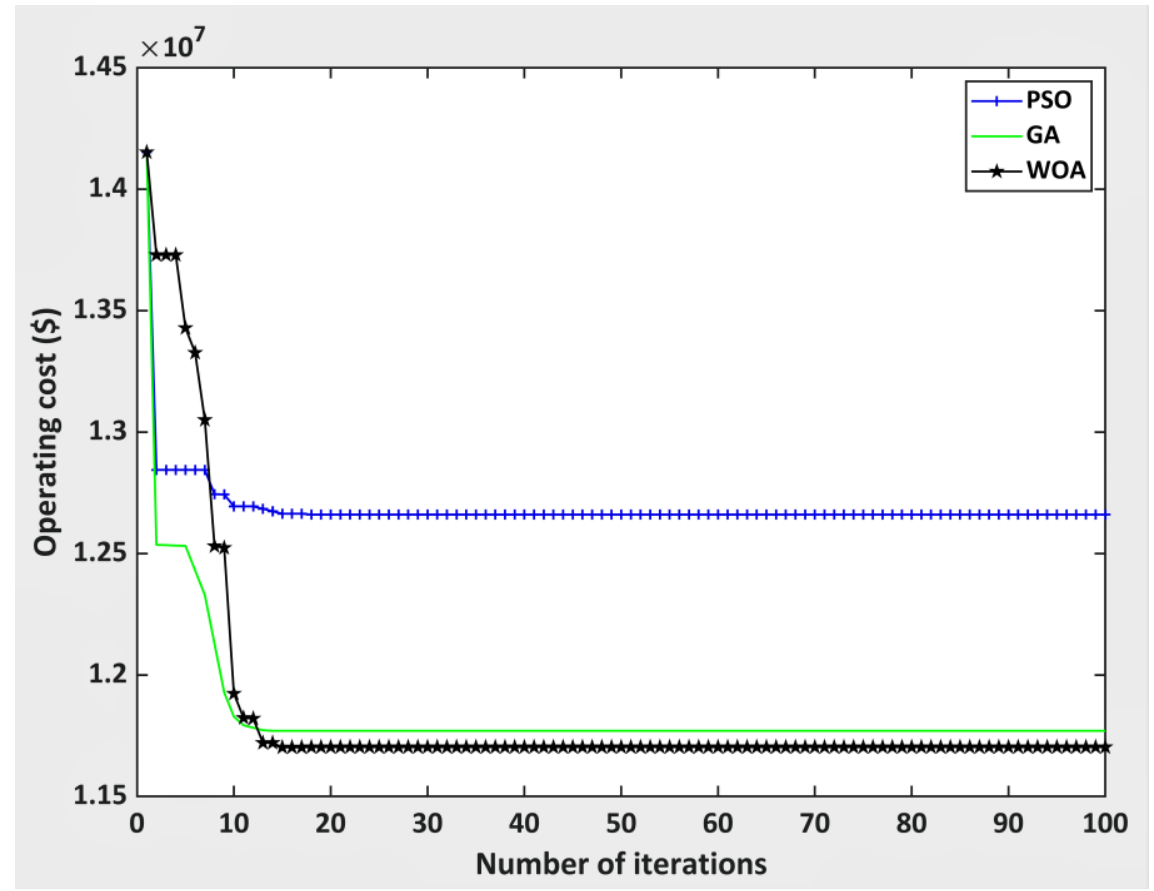

Figure 18. Change in operating cost with addition of FACTS at base-case using PSO GA and WOA. 


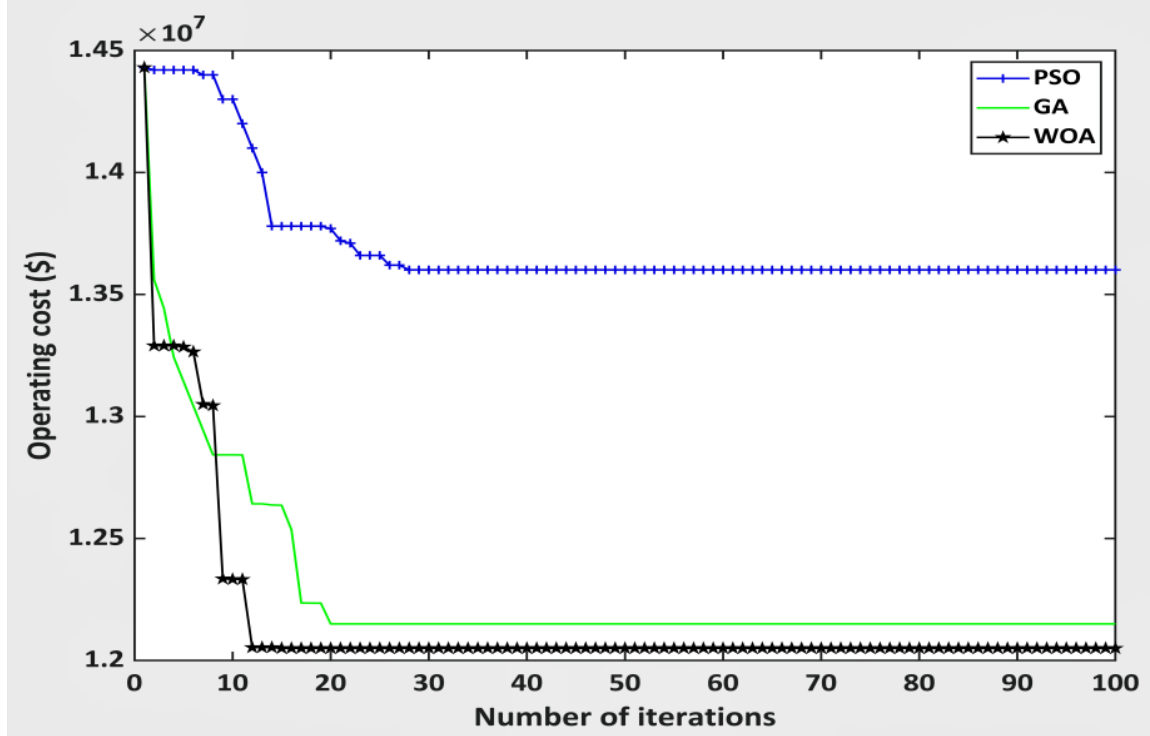

Figure 19. Change in operating cost with the addition of FACTS at $150 \%$ reactive loading using PSO, GA, and WOA.

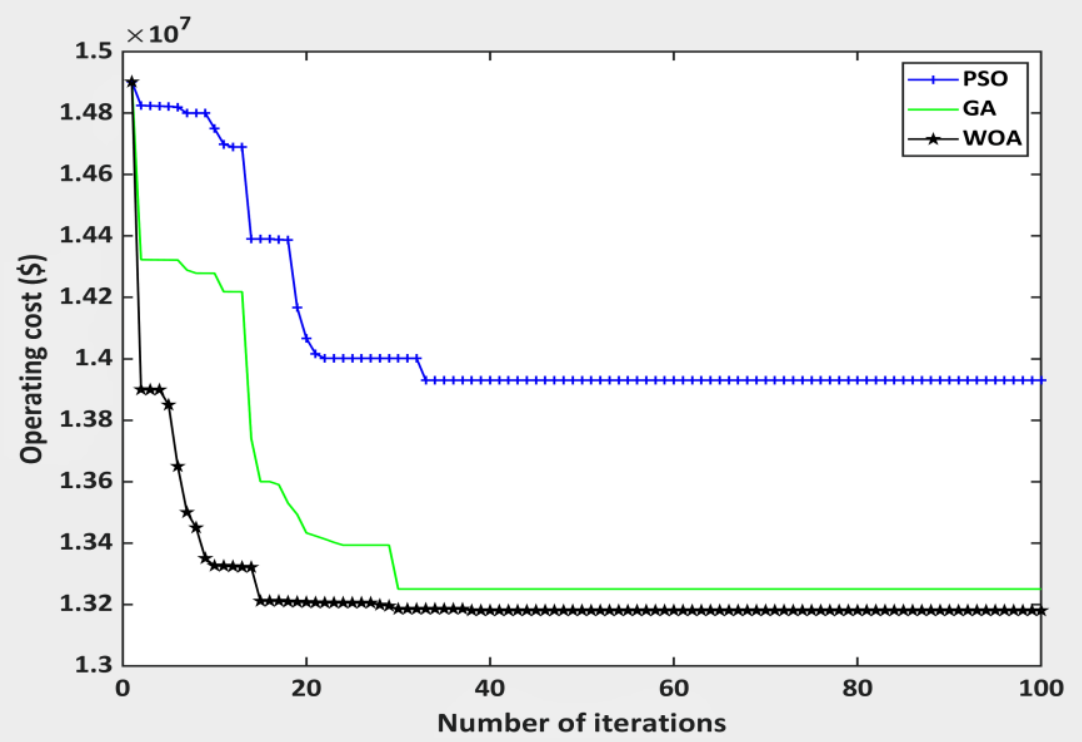

Figure 20. Change in the operating cost with the addition of FACTS at $200 \%$ reactive loading using PSO, GA, and WOA.

\section{Conclusions}

In this paper, a novel method was deployed for optimal placement and sizing of multiple types of FACTS devices as well as coordination with conventional reactive power sources. Initially, sensitivity analysis was carried out to find ideal locations for TCSC, UPFC, and SVC. Lines with a higher value of the Lmn index were considered weak lines and thus optimal locations for TCSC. Similarly, buses with a higher voltage deviation in response to increasing loading parameters are considered weak buses and suitable locations for SVC. Lines carrying higher active power are chosen as ideal for UPFC placement. After optimal placements of FACTS devices in the network, optimal settings of fitness function variables were determined by relatively newly introduced optimization techniques, namely whale optimization algorithm (WOA) and results were also compared with PSO and GA. The objective function included the cost of the active power loss and the total cost of FACTS devices. The proposed technique was 
applied to IEEE 14 and the 30 bus system. At 100\% reactive loading in the 14 bus system, WOA saved $\$ 719,000$ more as compared to PSO, and $\$ 125,000$ more as compared to GA. Similarly, at the base case for 30 bus systems, WOA saved $\$ 957,000$ and $\$ 67,000$ more as compared to PSO and GA, respectively. Hence WOA delivered better results than PSO and GA in terms of minimization of total operating cost and active power losses. It was also noted that net savings obtained using WOA were higher than those obtained using PSO and GA for all loading conditions.

Future work may involve the improvement of the whale optimization algorithm by hybridizing it with other metaheuristic techniques. Furthermore, as integration of renewable energy sources is increasing very rapidly in the power system, the optimal coordination of FACTS in the presence of renewable sources is worth exploring.

Author Contributions: M.N. gathered required data, conducted simulations, wrote the initial draft of the paper and updated it many times. K.I. supervised this research work, edited multiple drafts of paper, and coordinated with all authors. A.P. contributed by supervising and editing the paper. The rest of the authors reviewed drafts of the paper and added value by revisions. All authors have read and agreed to the published version of the manuscript.

Funding: This research benefited from funding received from USAID for exchange visit to ASU.

Acknowledgments: The authors are grateful to NUST and USAID for supporting this research work. We would also like to acknowledge support and guidance provided by Pooja Gupta and Reetam Sen Biswas in the School of Electrical Computer and Energy Engineering, Arizona State University (ASU), USA.

Conflicts of Interest: The authors declare no conflict of interest.

\section{References}

1. Liu, S.; Cruzat, C.; Kopsidas, K. Impact of transmission line overloads on network reliability and conductor ageing. In Proceedings of the 2017 IEEE, Manchester, UK, 18-22 June 2017; pp. 1-5.

2. Prabha, K. Power System Stability and Control, 1st ed.; Neal, J., Baja, M.G.L., Eds.; McGraw-Hill, Inc.: New York, NY, USA, 1994.

3. Aboreshaid, S.; Billinton, R. Probabilistic evaluation of voltage stability. IEEE Trans. Power Syst. 1999, 14, 342-348. [CrossRef]

4. Ghahremani, E.; Kamwa, I. Optimal Placement of Multiple-Type FACTS Devices to Maximize Power System Loadability Using a Generic Graphical User Interface. IEEE Trans. Power Syst. 2012, 1-15. [CrossRef]

5. Chansareewittaya, S.; Jirapong, P. Power transfer capability enhancement with optimal maximum number of facts controllers using evolutionary programming. In Proceedings of the IECON 2011-37th Annual Conference of the IEEE Industrial Electronics Society, Melbourne, VIC, Australia, 7-10 November 2011; pp. 4733-4738.

6. Tlijani, K.; Guesmi, T.; Hadj Abdallah, H. Optimal number, location and parameter setting of multiple TCSCs for security and system loadability enhancement. In Proceedings of the 10th International Multi-Conferences on Systems, Signals \& Devices 2013 (SSD13), Hammamet, Tunisia, 18-21 March 2013; pp. 1-6.

7. Sode-yome, A.; Mithulananthan, N.; Lee, K.Y. Reactive Power Loss Sensitivity Approach in Placing FACTS Devices and UPFC; IFAC: New York, NY, USA, 2004; Volume 45.

8. Faried, S.O.; Billinton, R.; Aboreshaid, S. Probabilistic technique for sizing FACTS devices for steady-state voltage profile enhancement. IET Gener. Transm. Distrib. 2009, 3, 385-392. [CrossRef]

9. Nascimento, S.D.; Gouvêa, M.M. Voltage Stability Enhancement in Power Systems with Automatic Facts Device Allocation. Energy Procedia 2017, 107, 60-67. [CrossRef]

10. Barrios-Martínez, E.; Ángeles-Camacho, C. Technical comparison of FACTS controllers in parallel connection. J. Appl. Res. Technol. 2017, 15, 36-44. [CrossRef]

11. Cai, L.J.; Erlich, I.; Stamtsis, G.C. Optimal choice and allocation of FACTS devices in deregulated electricity market using genetic algorithms. In Proceedings of the IEEE PES Power Systems Conference and Exposition, New York, NY, USA, 10-13 October 2004; pp. 201-207.

12. Zahid, M.; Chen, J.; Li, Y. Application of AAA for Optimized Placement of UPFC in Power systems. In Proceedings of the 2018 13th IEEE Conference on Industrial Electronics and Applications (ICIEA), Wuhan, China, 31 May-2 June 2018; pp. 30-35. 
13. Alharbi, F.T.; Almasabi, S.; Mitra, J. Enhancing Network Loadability Using Optimal TCSC Placement and Sizing. In Proceedings of the 2018 IEEE/PES Transmission and Distribution Conference and Exposition (T\&D), Denver, CO, USA, 16-19 April 2018; pp. 1-9.

14. Dawn, S.; Kumar Tiwari, P.; Kumar Goswami, A.; Panda, R. An approach for system risk assessment and mitigation by optimal operation of wind farm and FACTS devices in a centralized competitive power market. IEEE Trans. Sustain. Energy 2019, 10, 1054-1065. [CrossRef]

15. Dawn, S.; Tiwari, P.K.; Goswami, A.K. An approach for long term economic operations of competitive power market by optimal combined scheduling of wind turbines and FACTS controllers. Energy 2019, 181, 709-723. [CrossRef]

16. Hermanu, C.; Nizam, M.; Robbani, F.I. Optimal Placement of Unified Power Flow Controllers (UPFC) for Losses Reduction and Improve Voltage Stability Based on Sensitivity Analysis in 500 kV Java-Bali Electrical Power System. In Proceedings of the 2018 5th International Conference on Electric Vehicular Technology (ICEVT), Surakarta, Indonesia, 30-31 Octber 2018; pp. 83-87.

17. Jamnani, J.G.; Pandya, M. Coordination of SVC and TCSC for management of power flow by particle swarm optimization. Energy Procedia 2019, 156, 321-326. [CrossRef]

18. Magadum, R.B.; Dodamani, S.N.; Kulkarni, D.B. Optimal Placement of Unified Power Flow Controller (UPFC) using Fuzzy Logic. In Proceedings of the 2019 Fifth International Conference on Electrical Energy Systems (ICEES), Chennai, India, 21-22 February 2019; pp. 1-4.

19. Ziaee, O.; Choobineh, F.F. Thyristor-controlled switch capacitor placement in large-scale power systems via mixed integer linear programming and Taylor series expansion. In Proceedings of the 2014 IEEE PES General Meeting|Conference \& Exposition, National Harbor, MD, USA, 27-31 July 2014; p. 777.

20. Bhattacharyya, B.; Kumar, S. Approach for the solution of transmission congestion with multi-type FACTS devices. IET Gener. Transm. Distrib. 2016, 10, 2802-2809. [CrossRef]

21. Galvani, S.; Banna Sharifian, M.B.; Tarafdar Hagh, M. Unified power flow controller impact on power system predictability. IET Gener. Transm. Distrib. 2014, 8, 819-827. [CrossRef]

22. Inkollu, S.R.; Kota, V.R. Optimal setting of FACTS devices for voltage stability improvement using PSO adaptive GSA hybrid algorithm. Eng. Sci. Technol. Int. J. 2016, 19, 1166-1176. [CrossRef]

23. Mohammadi, A.; Jazaeri, M. A hybrid particle swarm optimization-genetic algorithm for optimal location of SVC devices in power system planning. In Proceedings of the 42nd International Universities Power Engineering Conference, Brighton, UK, 4-6 September 2007; pp. 1175-1181.

24. Kulkarni, P.P. Optimal Placement and Parameter setting of TCSC in Power Transmission System to increase the Power Transfer Capability. In Proceedings of the 2015 International Conference on Energy Systems and Applications (ICESA), Pune, India, 30 October-1 November 2015; pp. 735-739.

25. Khan, I.; Mallick, M.A.; Rafi, M.; Mirza, M.S. Optimal placement of FACTS controller scheme for enhancement of power system security in Indian scenario. J. Electr. Syst. Inf. Technol. 2015, 2, 161-171. [CrossRef]

26. Kavitha, K.; Neela, R. Optimal allocation of multi-type FACTS devices and its effect in enhancing system security using BBO, WIPSO \& PSO. J. Electr. Syst. Inf. Technol. 2017, 1-17.

27. Bhattacharyya, B.; Kumar, S. Electrical Power and Energy Systems Loadability enhancement with FACTS devices using gravitational search algorithm. Int. J. Electr. Power Energy Syst. 2016, 78, 470-479. [CrossRef]

28. Preedavichit, P.; Srivastava, S.C. Optimal reactive power dispatch considering FACTS devices. In Proceedings of the 1997 Fourth International Conference on Advances in Power System Control, Operation and Management, APSCOM-97 (Conf. Publ. No. 450), Hong Kong, China, 11-14 November 1997; pp. 620-625.

29. Raj, S.; Bhattacharyya, B. Optimal placement of TCSC and SVC for reactive power planning using Whale optimization algorithm. Swarm Evol. Comput. 2018, 40, 131-143. [CrossRef]

30. Bansal, H.O.; Agrawal, H.P.; Tiwana, S.; Singal, A.R.; Shrivastava, L. Optimal Location of FACT Devices to Control Reactive Power. Int. J. Eng. Sci. Technol. 2010, 2, 1556-1560.

31. Padhy, N.P.; Abdel-Rahim, A.M.M. Optimal Placement of Facts Devices for Practical Utilities. Int. J. Power Energy Syst. 2007, 27, 1-31. [CrossRef]

32. Narain, G.H. Understanding FACTs Concepts and Tehnology of Flexible AC Transmission Systems; Wiley-IEEE Press: New York, NY, USA, 2000; Volume 136.

33. Gyugyi, L. Unified power-flow control concept for flexible AC transmission systems. Gener. Transm. Distrib. IEE Proc. C 1992, 139, 323-331. [CrossRef] 
34. Masood, T.; Shah, S.K.; Tajammal, M.; Qureshi, S.A.; Khan, A.J.; Jameel, N.; Kothari, D.P. Unified power flow controller modeling and analysis technique on the GCC power grid. In Proceedings of the 2018 IEEE International Energy Conference (ENERGYCON), Limassol, Cyprus, 3-7 June 2018; pp. 1-6.

35. Bhattacharyya, B.; Gupta, V.K.; Kumar, S. UPFC with series and shunt FACTS controllers for the economic operation of a power system. Ain Shams Eng. J. 2014, 5, 775-787. [CrossRef]

36. Musirin, I.; Abdul Rahman, T.K. Novel fast voltage stability index (FVSI) for voltage stability analysis in power transmission system. In Proceedings of the Student Conference on Research and Development, Shah Alam, Malaysia, 17 July 2002; pp. 265-268.

37. Moghavvemi, M.; Faruque, O. Real-time contingency evaluation and ranking technique. IEE Proc.-Gener. Transm. Distrib. 2002, 145, 517. [CrossRef]

38. Moghavvemi, M.; Omar, F.M. Technique for contingency monitoring and voltage collapse prediction. IEE Proc.-Gener. Transm. Distrib. 1998, 145, 634. [CrossRef]

39. Ajjarapu, V.; Christy, C. The continuation power flow: A tool for steady state voltage stability analysis. IEEE Trans. Power Syst. 1992, 7, 416-423. [CrossRef]

40. Milano, F. An open source power system analysis toolbox. IEEE Trans. Power Syst. 2005, 20, 1199-1206. [CrossRef]

41. Cai, L.J.; Erlich, I. Optimal Choice and Allocation of FACTS Devices using Genetic Algorithms. Optim. Choice Alloc. FACTS Devices Using Genet. Algorithms 2004, 2004, 1-6.

42. Mirjalili, S.; Lewis, A. The Whale Optimization Algorithm. Adv. Eng. Softw. 2016, 95, 51-67. [CrossRef]

43. Zimmerman, R.; Murillo-Sánchez, C.E.; Thomas, R.J. MATPOWER: Steady-state operations, planning, and analysis tools for power systems research and education. IEEE Trans. Power Syst. 2011, 26, 12-19. [CrossRef]

44. Nasiri, J.; Khiyabani, F.M. A whale optimization algorithm (WOA) approach for clustering. Cogent Math. Stat. 2018, 5, 1-13. [CrossRef]

(C) 2020 by the authors. Licensee MDPI, Basel, Switzerland. This article is an open access article distributed under the terms and conditions of the Creative Commons Attribution (CC BY) license (http://creativecommons.org/licenses/by/4.0/). 